IHES/P $/ 05 / 25$

ULB-TH/05-18

\title{
Spin three gauge theory revisited
}

\author{
Xavier Bekaert ${ }^{a, 1}$, Nicolas Boulanger ${ }^{b, 2}$ and Sandrine Cnockaert ${ }^{c, 3}$ \\ ${ }^{a}$ Institut des Hautes Études Scientifiques \\ Le Bois-Marie, 35 route de Chartres, 91440 Bures-sur-Yvette (France) \\ ${ }^{b}$ Université de Mons-Hainaut, Mécanique et Gravitation \\ 6 avenue du Champ de Mars, 7000 Mons (Belgium) \\ ${ }^{c}$ Physique Théorique et Mathématique, Université Libre de Bruxelles \\ and International Solvay Institutes, \\ U.L.B. Campus Plaine, C.P. 231, B-1050, Bruxelles (Belgium)
}

\begin{abstract}
We study the problem of consistent interactions for spin-3 gauge fields in flat spacetime of arbitrary dimension $n>3$. Under the sole assumptions of Poincaré and parity invariance, local and perturbative deformation of the free theory, we determine all nontrivial consistent deformations of the abelian gauge algebra and classify the corresponding deformations of the quadratic action, at first order in the deformation parameter. We prove that all such vertices are cubic, contain a total of either three or five derivatives and are uniquely characterized by a rank-three constant tensor (an internal algebra structure constant). The covariant cubic vertex containing three derivatives is the vertex discovered by Berends, Burgers and van Dam, which however leads to inconsistencies at second order in the deformation parameter. In dimensions $n>4$ and for a completely antisymmetric structure constant tensor, another covariant cubic vertex exists, which contains five derivatives and passes the consistency test where the previous vertex failed.
\end{abstract}

\footnotetext{
${ }^{1}$ E-mail address: bekaert@ihes.fr

${ }^{2}$ Chargé de Recherches FNRS (Belgium); nicolas.boulanger@umh.ac.be

${ }^{3}$ Aspirant du FNRS (Belgium); sandrine.cnockaert@ulb.ac.be
} 


\section{Introduction}

Whereas gauge theories describing free massless fields of arbitrary high spin are by now well established, it still remains unclear whether nontrivial consistent self-couplings and/or crosscouplings among those fields may exist at the level of the action, such that the deformed gauge algebra is non-abelian. The old Fronsdal programme of introducing consistent couplings among higher-spin gauge fields [1] is still far away from completion. Actually, there is a general belief that such interactions are forbidden, except perhaps when the cosmological constant is nonvanishing, in which case encouraging results have been found at the level of equations of motion (see e.g. [2,3] and references therein).

The Fronsdal programme was initially investigated in two distinct directions: either searching for consistent vertices for higher-spin gauge fields interacting with each other but not with gravity, or attempting to couple consistently some given higher-spin gauge field with gravity. On the one hand, the problem of consistent interactions among higher-spin gauge fields in Minkowski spacetime $\mathbb{R}^{n-1,1}$ was addressed in [4-15] where some positive results have been obtained. In the light-cone gauge, three-point couplings between completely symmetric $^{4}$ gauge fields with arbitrary spins $s>2$, were constructed in $[5,13,15]$. For the pure spin-3 case, a cubic vertex was obtained in a covariant form by Berends, Burgers and van Dam [6]. These results describe consistent interactions at first order in a deformation parameter $g$ and involve higher-derivatives. However, no-go results soon demonstrated the impossibility of extending these interactions to the next orders in powers of $g$ for the pure spin-3 case $[7,9,10]$. On the other hand, the first explicit attempts to introduce interactions between higher-spin gauge fields and gravity encountered severe problems [17].

Very early, the idea was proposed that a consistent higher-spin gauge theory could exist, provided all spins are taken into account [1]. In order to overcome the gravitational coupling problem, it was also suggested to perturb around a curved, conformally-flat background, like for example $A d S_{n}$. In such a case, the cosmological constant $\Lambda$ can be used to cancel the positive mass dimensions appearing with the increasingly many derivatives of the vertices. As the works of Fradkin, Vasiliev and others show, interesting results have indeed been obtained in those directions, even at the level of the action [18].

If there is a lesson to learn from decades of efforts toward a consistent theory of interacting higher-spin gauge fields, it certainly is the unusual character of the possible interactions. For instance, the cubic vertices contain more than two derivatives. ${ }^{5}$ This, in turn, can be linked to the fact that the spin-s curvature is expressed via $s$ derivatives of the gauge field

\footnotetext{
${ }^{4}$ Light-cone cubic vertices involving mixed symmetry gauge fields were computed in dimensions $n=5,6$ [16].

${ }^{5}$ The full theory presented in [2] is even expected to be non-local.
} 
$[4,19]$. Consequently, in order to investigate further the possible local higher-spin consistent interactions, it is of prime importance to use as general a tool as possible. A cohomological method is known [20], which offers all the generality one could wish and clearly organizes the calculation of the nontrivial consistent couplings. In this approach, the old Noether method (see for instance [9]) is reformulated in the BRST framework where consistent couplings define deformations of the solution of the master equation. This formulation has been used recently in different contexts (see e.g. [21-23] and references therein).

In the present paper, we come back to the initial (and more modest) problem of consistent interactions among higher-spin gauge fields in flat spacetime and concentrate on the pure spin-3 case. The motivation behind our work is the existence of the new method [20] developed in the meantime, which allows for an exhaustive treatment of the consistent interaction problem while, in the aforementioned works [5-11,13-15], classes of deformation candidates were rejected $a b$ initio from the analysis for the sake of simplicity. For example, spin-3 cubic vertices containing more than 3 derivatives were not considered in the otherwise very general analysis of [6]. This ansatz was too restrictive since another cubic vertex with five derivatives exists in dimensions higher than four (it is written explicitly in Appendix B). Moreover, without fixing a priori the maximal number of derivatives, we show that vertices deforming the gauge algebra must contain a total number of either three or five derivatives. ${ }^{6}$

The paper is organized as follows. In Section 2, we review the free theory of massless spin-3 gauge fields represented by completely symmetric rank-3 tensors. Our principal hypotheses are spelled out in Section 3.1 and our main results are collected in Theorems 1 and 2 presented in Section 3.2. The section 4 gathers together the main BRST results needed for the exhaustive treatment of the interaction problem: The BRST spectrum of the theory is presented in Section 4.1. Some cohomological results have already been obtained in [24], such as the cohomology $H^{*}(\gamma)$ of the gauge differential $\gamma$ and the so called characteristic cohomology $H_{k}^{n}(\delta \mid d)$ in antighost number $k \geqslant 2$. We recall the content of these groups in Sections 4.3 and 4.5. The calculation of the invariant characteristic cohomology $H_{k}^{n}(\delta \mid d, H(\gamma))$ constitutes the core of the BRST analysis and is achieved in Section 4.6. The self-interaction question is answered in Section 5. We give our conclusions and discuss several directions for future research in Section 6.

\footnotetext{
${ }^{6}$ This result is in agreement with the general upper bound $k<s_{1}+s_{2}+s_{3}$ on the total number $k$ of derivatives in a cubic vertex containing completely symmetric fields of respective spin $s_{1}, s_{2}$ and $s_{3}$ [15].
} 


\section{Free theory}

The local action for a collection $\left\{h_{\mu \nu \rho}^{a}\right\}$ of $N$ non-interacting completely symmetric massless spin-3 gauge fields in flat spacetime is [1]

$$
\begin{aligned}
S_{0}\left[h_{\mu \nu \rho}^{a}\right]=\sum_{a=1}^{N} \int d^{n} x \quad[ & -\frac{1}{2} \partial_{\sigma} h_{\mu \nu \rho}^{a} \partial^{\sigma} h^{a \mu \nu \rho}+\frac{3}{2} \partial^{\mu} h_{\mu \rho \sigma}^{a} \partial_{\nu} h^{a \nu \rho \sigma}+ \\
& \left.\frac{3}{2} \partial_{\mu} h_{\nu}^{a} \partial^{\mu} h^{a \nu}+\frac{3}{4} \partial_{\mu} h^{a \mu} \partial_{\nu} h^{a \nu}-3 \partial_{\mu} h_{\nu}^{a} \partial_{\rho} h^{a \rho \mu \nu}\right],
\end{aligned}
$$

where $h_{\mu}^{a}:=\eta^{\nu \rho} h_{\mu \nu \rho}^{a}$. The Latin indices are internal indices taking $N$ values. They are raised and lowered with the Kronecker delta's $\delta^{a b}$ and $\delta_{a b}$. The Greek indices are space-time indices taking $n$ values, which are lowered (resp. raised) with the "mostly plus" Minkowski metric $\eta_{\mu \nu}$ (resp. $\left.\eta^{\mu \nu}\right)$.

The action (2.1) is invariant under the gauge transformations

$$
\delta_{\lambda} h_{\mu \nu \rho}^{a}=3 \partial_{(\mu} \lambda_{\nu \rho)}^{a}, \quad \eta^{\mu \nu} \lambda_{\mu \nu}^{a} \equiv 0
$$

where the gauge parameters $\lambda_{\nu \rho}^{a}$ are symmetric and traceless ${ }^{7}$. Curved (resp. square) brackets on spacetime indices denote strength-one complete symmetrization (resp. antisymmetrization) of the indices. The gauge transformations (2.2) are abelian and irreducible.

The field equations read

$$
\frac{\delta S_{0}}{\delta h_{\mu \nu \rho}^{a}} \equiv G_{a}^{\mu \nu \rho}=0
$$

where

$$
G_{\mu \nu \rho}^{a}:=F_{\mu \nu \rho}^{a}-\frac{3}{2} \eta_{(\mu \nu} F_{\rho)}^{a}
$$

is the "Einstein" tensor and $F_{\mu \nu \rho}^{a}$ the Fronsdal (or "Ricci") tensor

$$
F_{\mu \nu \rho}^{a}:=\square h_{\mu \nu \rho}^{a}-3 \partial^{\sigma} \partial_{(\mu} h_{\nu \rho) \sigma}^{a}+3 \partial_{(\mu} \partial_{\nu} h_{\rho)}^{a} .
$$

The Fronsdal tensor is gauge invariant thanks to the tracelessness of the gauge parameters. Because we have $\delta_{\lambda} S_{0}\left[h_{\mu \nu \rho}^{a}\right]=0$ for the gauge transformations $(2.2)$, the Einstein tensor $G_{\mu \nu \rho}^{a}$ satisfies the Noether identities

$$
\partial^{\rho} G_{\mu \nu \rho}^{a}-\frac{1}{n} \eta_{\mu \nu} \partial^{\rho} G_{\rho}^{a} \equiv 0 \quad\left(G_{\rho}^{a}:=\eta^{\mu \nu} G_{\mu \nu \rho}^{a}\right)
$$

\footnotetext{
${ }^{7}$ Quadratic non-local actions [25] have been proposed in order to get rid of the trace constraint (2.2) on the gauge parameter. Since locality is an important hypothesis of the present work, we do not discuss the non-local formulation here. Notice that by introducing a pure gauge field (sometimes refered to as "compensator"), it is possible to write a local (but higher-derivative) action for spin-3 [25] that is invariant under unconstrained gauge transformations. Very recently, this action was generalized to the arbitrary spin- $s$ case by further adding an auxiliary field [26] (see also [27] for an older "non-minimal" version of it).
} 
related to the symmetries of the gauge parameters $\lambda_{\mu \nu}^{a}$; in other words, the 1.h.s. of (2.6) is symmetric and traceless.

The gauge symmetries enable one to get rid of some components of $h_{\mu \nu \rho}^{a}$, leaving it onshell with $N_{3}^{n}$ independent physical components, where $N_{3}^{n}$ is the dimension of the irreducible representation of the "little group" $O(n-2)(n \geqslant 3)$ corresponding to a completely symmetric rank 3 traceless tensor in dimension $n-2$. One has $N_{3}^{n}=\frac{n^{3}-3 n^{2}-4 n+12}{6}$. Of course, $N_{3}^{4}=2$ for the two helicity states \pm 3 in dimension $n=4$. Note also that there is no propagating physical degree of freedom in $n=3$ since $N_{3}^{3}=0$, so that we restrict our present work to $n>3$.

An important object is the curvature (or "Riemann") tensor [4, 19, 28]

$$
K_{\alpha \mu|\beta \nu| \gamma \rho}^{a}:=8 \partial_{[\gamma} \partial_{[\beta} \partial_{[\alpha} h_{\mu] \nu] \rho]}^{a}
$$

which is antisymmetric in $\alpha \mu, \beta \nu, \gamma \rho$ and invariant under gauge transformations (2.2), where the gauge parameters $\lambda_{\mu \nu}^{a}$ are however not necessarily traceless.

Its importance, apart from gauge invariance with unconstrained gauge parameters, stems from the fact that the field equations (2.3) are equivalent ${ }^{8}$ to the following equations

$$
\eta^{\alpha \beta} K_{\alpha \mu|\beta \nu| \gamma \rho}^{a}=0
$$

This was proved in the work [29] by combining various former results $[25,28,30]$.

\section{Deformations of the free theory}

\subsection{Basic assumptions}

We assume, as in the traditional Noether deformation procedure, that the deformed action can be expressed as a power series in a coupling constant $g$, the zeroth-order term in the expansion describing the free theory $S_{0}$ :

$$
S=S_{0}+g S_{1}+\mathcal{O}\left(g^{2}\right)
$$

The procedure is then perturbative: one tries to construct the deformations order by order in the deformation parameter $g$.

Some physical requirements naturally come out:

- Poincaré and parity symmetry: We ask that the deformed Lagrangian be invariant under the Poincaré group. Therefore, it should not depend explicitly on the space-time

\footnotetext{
${ }^{8}$ As usual in field theory, we work in a space of smooth functions that vanish at infinity. In particular, polynomials in $x^{\mu}$ are forbidden.
} 
cartesian coordinates $\left\{x^{\mu}\right\}$. The Lagrangian is moreover required to be invariant under the parity transformation. This implies that all Greek indices have to be contracted by means of the Minkowski metric only.

- Nontriviality: We reject trivial deformations arising from field-redefinitions that reduce to the identity at order $g^{0}$ :

$$
\phi \longrightarrow \phi^{\prime}=\phi+g \varphi(\phi, \partial \phi, \cdots)+\mathcal{O}\left(g^{2}\right)
$$

- Consistency: A deformation of a theory is called consistent if the deformed theory possesses the same number of (possibly deformed) independent gauge symmetries, reducibility identities, etc., as the system we started with. In other words, the number of physical degrees of freedom is unchanged.

- Locality: The deformed action $S[\phi]$ must be a local functional. The deformations of the gauge transformations, etc., must be local functions, as well as the allowed field redefinitions.

We remind the reader that a local function of some set of fields $\phi^{i}$ is a smooth function of the fields $\phi^{i}$ and their derivatives $\partial \phi^{i}, \partial^{2} \phi^{i}, \ldots$ up to some finite order, say $k$, in the number of derivatives. Such a set of variables $\phi^{i}, \partial \phi^{i}, \ldots, \partial^{k} \phi^{i}$ will be collectively denoted by [ $\left.\phi^{i}\right]$. Therefore, a local function of $\phi^{i}$ is denoted by $f\left(\left[\phi^{i}\right]\right)$. A local $p$-form $(0 \leqslant p \leqslant n)$ is a differential $p$-form the components of which are local functions:

$$
\omega=\frac{1}{p !} \omega_{\mu_{1} \ldots \mu_{p}}\left(x,\left[\phi^{i}\right]\right) d x^{\mu_{1}} \wedge \cdots \wedge d x^{\mu_{p}} .
$$

A local functional is the integral of a local $n$-form.

\subsection{Main results}

Theorems 1 and 2 are presented in this Section. They constitute strong yes-go and no-go theorems that generalize previous works on spin-3 self-interactions.

Theorem 1. Let $h_{\mu \nu \rho}^{a}$ be a collection of spin-3 gauge fields $(a=1, \ldots, N)$ described by the local and quadratic action of Fronsdal, in dimension $n>3$.

At first order in some smooth deformation parameter, the nontrivial consistent local deformations of the (abelian) gauge algebra that are invariant under parity and Poincaré transformations, may always be assumed to be closed off-shell and are in one-to-one correspondence with the structure constant tensors

$$
C^{a}{ }_{b c}=-C^{a}{ }_{c b}
$$


of an anticommutative internal algebra, that may be taken as deformation parameters.

Moreover, the most general gauge transformations deforming the gauge algebra at first order in $C=(f, g)$ are equal to

$$
\delta_{\lambda} h_{\mu \nu \rho}^{a}=3 \partial_{(\mu} \lambda_{\nu \rho)}^{a}+f_{b c}^{a} \Phi_{\mu \nu \rho}^{b c}+g_{b c}^{a}\left(\Psi_{\mu \nu \rho}^{b c}-\frac{1}{n} \eta_{(\mu \nu} \Psi_{\rho)}^{b c}\right)+\mathcal{O}\left(C^{2}\right)
$$

up to gauge transformations that either are trivial or do not deform the gauge algebra at first order, where $\Phi_{\mu \nu \rho}^{b c}$ and $\Psi_{\mu \nu \rho}^{b c}$ are bilinear local functions of the gauge field $h_{\mu \nu \rho}^{a}$ and the traceless gauge parameter $\lambda_{\mu \nu}^{a}$. The expression for $\Phi$ is lengthy and thus given in the appendix A, while

$$
\Psi_{\mu \nu \rho}^{b c}=-\frac{1}{3} \eta^{\alpha \beta} \partial_{[\mu} h_{\alpha] \nu[\sigma, \tau]}^{b} \partial_{[\rho} \lambda_{\beta]}^{c \sigma, \tau}+\text { perms }
$$

where a coma denotes a partial derivative and "perms" stands for the sum of terms obtained via all nontrivial permutations of the indices $\mu, \nu, \rho$ from the first term of the r.h.s.

The structure constant tensors $f_{b c}^{a}$ and $g_{b c}^{a}$ are some arbitrary constant tensors that are antisymmetric in the indices bc. In mass units, the coupling constant $f_{b c}^{a}$ has dimension $-n / 2$ and $g_{b c}^{a}$ has dimension $-2-n / 2$.

Both of these deformations exist in any dimension $n \geqslant 5$. In the case $n=4$, the structure constant tensor $g_{b c}^{a}$ vanishes.

Firstly, we found a deformation of the gauge symmetries (the one corresponding to the coefficients $g_{b c}^{a}$ ) which had not been written explicitly in previous spin-3 analyzes in flat space-time. Secondly, without imposing any restriction on the maximal number of derivatives (as was implicit in most former works) we prove that the allowed possibilities are extremely restricted.

An important question is whether these algebra deformations can be obtained from an appropriate flat space-time limit of the $(A) d S_{n}$ higher-spin algebras containing a finitedimensional non-Abelian internal subalgebra (studied in details by Vasiliev and collaborators [31]). An indication that this might be the case is provided by the deformation of the gauge transformations Eq. (3.10) involving the tensor $\Psi_{\mu \nu \rho}^{a b}$. The presence of the term $\partial_{[\mu} h_{\alpha] \nu[\sigma, \tau]}^{b}$ in (3.11) is reminiscent of the second frame-like connection (see e.g. the second reference of [3]). They both involve two derivatives of the spin-3 field and have the $g l(n)$-symmetry

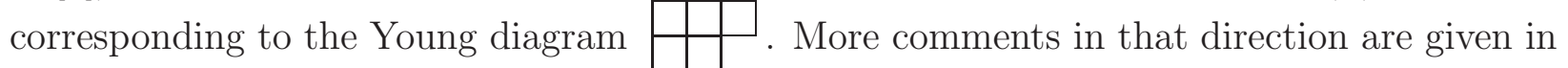
sections 4.3 and 5.3 .

Another important physical question is whether or not these first-order gauge symmetry deformations possess some Lagrangian counterpart, i.e. if there exist vertices that are invariant under (3.10) at first order in $C$. The following theorem provides a sufficient condition for that:

\footnotetext{
${ }^{9}$ For example $\Phi_{, \alpha}^{i} \equiv \partial_{\alpha} \Phi^{i}$.
} 
Theorem 2. Let the constant tensor $C_{a b c}=\left(f_{a b c}, g_{a b c}\right)$ be completely antisymmetric, where $C_{a b c}:=\delta_{a d} C_{b c}^{d}$. Then,

- The quadratic local action (2.1) in dimension $n>3$ admits a first-order consistent deformation

$$
S\left[h_{\mu \nu \rho}^{a}\right]=S_{0}+f_{a b c} S^{a b c}+g_{a b c} T^{a b c}+\mathcal{O}\left(C^{2}\right)
$$

which is gauge invariant under the deformed gauge transformations (3.10) at first order in the deformation parameters. Furthermore, this antisymmetry condition on the tensor $f_{b c}^{a}$ is necessary for the existence of the corresponding deformation of the action.

- The vertices in the first-order deformations are determined uniquely by the structure constants $f_{a b c}$ and $g_{a b c}$, modulo vertices that do not deform the gauge algebra. The corresponding local functionals $S^{a b c}\left[h_{\mu \nu \rho}^{d}\right]$ and $T^{a b c}\left[h_{\mu \nu \rho}^{d}\right]$ are cubic in the gauge field and respectively contain three and five derivatives. Actually, there are no other nontrivial consistent vertices containing at most three derivatives that deform the gauge transformation at first order.

- At second order in $C$, the deformation of the gauge algebra can be assumed to close off-shell without loss of generality, but it is obstructed if and only if $f_{a b c} \neq 0$.

The first-order covariant cubic deformation $S_{a}^{b c}\left[h_{\mu \nu \rho}^{d}\right]$ is the Berends-Burgers-van Dam vertex [6] (reviewed for completeness in Appendix A) while the other cubic deformation $T_{a}^{b c}\left[h_{\mu \nu \rho}^{d}\right]$ is written in Appendix B. We do not know yet if the antisymmetry condition on the structure constant $g_{b c}^{a}$ is necessary or not for the existence of a consistent vertex at first order.

It is possible to provide a more intrinsic characterization of the conditions on the constant tensors. Let $\mathcal{A}$ be an anticommutative algebra of dimension $N$ with a basis $\left\{T_{a}\right\}$. Its multiplication law $*: \mathcal{A}^{2} \rightarrow \mathcal{A}$ obeys $a * b=-b * a$ for any $a, b \in \mathcal{A}$, which is equivalent to the fact that the structure constant tensor $C^{a}{ }_{b c}$ defined by $T_{b} * T_{c}=C^{a}{ }_{b c} T_{a}$ is antisymmetric in the covariant indices: $C^{a}{ }_{b c}=-C^{a}{ }_{c b}$. Moreover, let us assume that the algebra $\mathcal{A}$ is a Euclidean space, i.e. it is endowed with a scalar product $\langle\rangle:, \mathcal{A}^{2} \rightarrow \mathbb{R}$ with respect to which the basis $\left\{T_{a}\right\}$ is orthonormal, $\left\langle T_{a}, T_{b}\right\rangle=\delta_{a b}$. For an anticommutative algebra, the scalar product is said to be invariant (under the left or right multiplication) if and only if $\langle a * b, c\rangle=\langle a, b * c\rangle$ for any $a, b, c \in \mathcal{A}$, and the latter property is equivalent to the complete antisymmetry of the trilinear form

$$
C: \mathcal{A}^{3} \rightarrow \mathbb{R}:(a, b, c) \mapsto C(a, b, c)=\langle a, b * c\rangle
$$

or, in components, to the complete antisymmetry property of the covariant tensor $C_{a b c}:=$ $\delta_{a d} C_{b c}^{d}$. 
The gauge algebra inferred from the Berends-Burgers-van Dam vertex is inconsistent at second order [7,9] and no corresponding quartic interaction can be constructed [10]. Originally, consistency of the Berends-Burgers-van Dam deformation at second order was shown to require that $f_{e c}^{d} f_{a b}^{e}=f_{a e}^{d} f_{b c}^{e}[9]$, which means that the corresponding internal algebra is associative $(a * b) * c=a *(b * c)$. In Section 5.2.2, we actually obtain a stronger condition from consistency: $f_{e c}^{d} f_{a b}^{e}=0$, i.e. the internal algebra is nilpotent of order three: $(a * b) * c=0$. In any case, to derive that the Berends-Burgers-van Dam vertex is inconsistent at order two, one may use the following well-known lemma

Lemma 1. If an anticommutative algebra endowed with an invariant scalar product is associative, then the product of any two elements is zero (in other words, the algebra is nilpotent of order two).

Proof: Under the hypotheses of Lemma 1, one gets $\langle a * b, b * a\rangle=\langle a, b *(b * a)\rangle=$ $\langle a,(b * b) * a\rangle=0$ which implies $a * b=0$ for any $a, b \in \mathcal{A}$.

An exciting result is that the second deformation corresponding to $g_{a b c}=g_{[a b c]}$ passes the gauge algebra consistency requirement where the vertex of Berends, Burgers and van Dam fails. Unfortunately, we do not know if there exist second order gauge transformations that are consistent at this order.

The proofs of Theorems 1 and 2 are given in Section 5. They rely on a BRST cohomological reformulation presented in the next Section.

\section{BRST settings}

\subsection{BRST spectrum and differential}

According to the general rules of the BRST-antifield formalism, a grassmann-odd ghost $C_{\mu \nu}^{a}$ is introduced, which accompanies each grassmann-even gauge parameter $\lambda_{\mu \nu}^{a}$. In particular, it possesses the same algebraic symmetries as $\lambda_{\mu \nu}^{a}$ : it is symmetric and traceless in its spacetime indices. Then, to each field and ghost of the spectrum, a corresponding antifield (or antighost) is added, with the same algebraic symmetries but the opposite Grassmann parity. A $\mathbb{Z}$-grading called ghost number $(g h)$ is associated with the BRST differential $s$, while the antighost number (antigh) of the antifield $Z^{*}$ associated with the field (or ghost) $Z$ is given by antigh $\left(Z^{*}\right) \equiv g h(Z)+1$. More precisely, in the theory under consideration, the spectrum of fields (including ghosts) and antifields together with their respective ghost and antighost numbers is given by

- the fields $h_{\mu \nu \rho}^{a}$, with ghost number 0 and antighost number 0 ; 
- the ghosts $C_{\mu \nu}^{a}$, with ghost number 1 and antighost number 0 ;

- the antifields $h_{a}^{* \mu \nu \rho}$, with ghost number -1 and antighost number 1 ;

- the antifields $C_{a}^{* \mu \nu}$, with ghost number -2 and antighost number 2 .

The BRST differential $s$ of the free theory (2.1), (2.2) is generated by the functional

$$
W_{0}=S_{0}\left[h^{a}\right]+\int d^{n} x\left(3 h_{a}^{* \mu \nu \rho} \partial_{\mu} C_{\nu \rho}^{a}\right) .
$$

More precisely, $W_{0}$ is the generator of the BRST differential $s$ of the free theory through

$$
s A=\left(W_{0}, A\right)_{a . b .}
$$

where the antibracket $(,)_{a . b}$. is defined by

$$
(A, B)_{a . b .}=\frac{\delta^{R} A}{\delta \Phi^{I}} \frac{\delta^{L} B}{\delta \Phi_{I}^{*}}-\frac{\delta^{R} A}{\delta \Phi_{I}^{*}} \frac{\delta^{L} B}{\delta \Phi^{I}} .
$$

The functional $W_{0}$ is a solution of the master equation

$$
\left(W_{0}, W_{0}\right)_{a . b .}=0
$$

In the theory at hand, the BRST-differential $s$ decomposes into $s=\gamma+\delta$. The first piece $\gamma$, the differential along the gauge orbits, is associated with another grading called pureghost number (puregh) and increases it by one unit, whereas the Koszul-Tate differential $\delta$ decreases the antighost (or antifield) number by one unit. The differential $s$ increases the ghost number by one unit. Furthermore, the ghost, antighost and pureghost gradings are not independent. We have the relation

$$
g h=\text { puregh }- \text { antigh } .
$$

The pureghost number, antighost number, ghost number and grassmannian parity of the various fields are displayed in Table 1.

\begin{tabular}{|c|c|c|c|c|}
\hline $\mathrm{Z}$ & puregh $(Z)$ & antigh $(Z)$ & $g h(Z)$ & parity $(\bmod 2)$ \\
\hline$h_{\mu \nu \rho}^{a}$ & 0 & 0 & 0 & 0 \\
$C_{\mu \nu}^{a}$ & 1 & 0 & 1 & 1 \\
$h_{a}^{* \mu \nu \rho}$ & 0 & 1 & -1 & 1 \\
$C_{a}^{* \mu \nu}$ & 0 & 2 & -2 & 0 \\
\hline
\end{tabular}

Table 1: pureghost number, antighost number, ghost number and parity of the (anti)fields. 
The action of the differentials $\delta$ and $\gamma$ gives zero on all the fields of the formalism except in the few following cases:

$$
\begin{aligned}
\delta h_{a}^{* \mu \nu \rho} & =G_{a}^{\mu \nu \rho}, \\
\delta C_{a}^{* \mu \nu} & =-3\left(\partial_{\rho} h_{a}^{* \mu \nu \rho}-\frac{1}{n} \eta^{\mu \nu} \partial_{\rho} h_{a}^{* \rho}\right), \\
\gamma h_{\mu \nu \rho}^{a} & =3 \partial_{(\mu} C_{\nu \rho)}^{a} .
\end{aligned}
$$

\subsection{BRST deformation}

As shown in [20], the Noether procedure can be reformulated within a BRST-cohomological framework. Any consistent deformation of the gauge theory corresponds to a solution

$$
W=W_{0}+g W_{1}+g^{2} W_{2}+\mathcal{O}\left(g^{3}\right)
$$

of the deformed master equation $(W, W)_{a . b .}=0$. Consequently, the first-order nontrivial consistent local deformations $W_{1}=\int a^{n, 0}$ are in one-to-one correspondence with elements of the cohomology $H^{n, 0}(s \mid d)$ of the zeroth order BRST differential $s=\left(W_{0}, \cdot\right)$ modulo the total derivative $d$, in maximum form-degree $n$ and in ghost number 0 . That is, one must compute the general solution of the cocycle condition

$$
s a^{n, 0}+d b^{n-1,1}=0
$$

where $a^{n, 0}$ is a top-form of ghost number zero and $b^{n-1,1}$ a $(n-1)$-form of ghost number one, with the understanding that two solutions of (4.17) that differ by a trivial solution should be identified

$$
a^{n, 0} \sim a^{n, 0}+s p^{n,-1}+d q^{n-1,0}
$$

as they define the same interactions up to field redefinitions (3.9). The cocycles and coboundaries $a, b, p, q, \ldots$ are local forms of the field variables (including ghosts and antifields).

The corresponding second-order interactions $W_{2}$ must satisfy the consistency condition

$$
s W_{2}=-\frac{1}{2}\left(W_{1}, W_{1}\right)_{a . b .} .
$$

This condition is controlled by the local BRST cohomology group $H^{n, 1}(s \mid d)$.

\subsection{Cohomology of $\gamma$}

In the context of local free theories in Minkowski space for massless spin-s gauge fields represented by completely symmetric (and double traceless when $s>3$ ) rank $s$ tensors, 
the groups $H^{*}(\gamma)$ have recently been calculated [24]. Accordingly, we only recall the latter results in the special case $s=3$ and introduce some new notations.

Proposition 1. The cohomology of $\gamma$ is isomorphic to the space of functions depending on

- the antifields $h_{a}^{* \mu \nu \rho}, C_{a}^{* \mu \nu}$ and their derivatives, denoted by $\left[\Phi^{* i}\right]$,

- the curvature and its derivatives $\left[K_{\alpha \mu|\beta \nu| \gamma \rho}^{a}\right]$,

- the symmetrized derivatives $\partial_{\left(\alpha_{1}\right.} \ldots \partial_{\alpha_{k}} F_{\mu \nu \rho)}^{a}$ of the Fronsdal tensor,

- the ghosts $C_{\mu \nu}^{a}$ and the traceless parts of $\partial_{[\alpha} C_{\mu] \nu}^{a}$ and $\partial_{[\alpha} C_{\mu][\nu, \beta]}^{a}$.

Thus, identifying with zero any $\gamma$-exact term in $H(\gamma)$, we have

$$
\gamma f=0
$$

if and only if

$$
f=f\left(\left[\Phi^{* i}\right],\left[K_{\alpha \mu|\beta \nu| \gamma \rho}^{a}\right],\left\{F_{\mu \nu \rho}^{a}\right\}, C_{\mu \nu}^{a}, \widehat{T}_{\alpha \mu \mid \nu}^{a}, \widehat{U}_{\alpha \mu \mid \beta \nu}^{a}\right)
$$

where $\left\{F_{\mu \nu \rho}^{a}\right\}$ stands for the completely symmetrized derivatives $\partial_{\left(\alpha_{1}\right.} \ldots \partial_{\alpha_{k}} F_{\mu \nu \rho)}^{a}$ of the Fronsdal tensor, while $\widehat{T}_{\alpha \mu \mid \nu}^{a}$ denotes the traceless part of $T_{\alpha \mu \mid \nu}^{a}:=\partial_{[\alpha} C_{\mu] \nu}^{a}$ and $\widehat{U}_{\alpha \mu \mid \beta \nu}^{a}$ the traceless part of $U_{\alpha \mu \mid \beta \nu}^{a}:=\partial_{[\alpha} C_{\mu][\nu, \beta]}^{a}$.

This proposition provides the possibility of writing down the most general gauge-invariant interaction terms. Such higher-derivative Born-Infeld-like Lagrangians were already considered in Ref. [12]. These deformations are consistent to all orders but they do not deform the gauge transformations (2.2). Also notice that any function of the Fronsdal tensor or its derivatives corresponds to a field redefinition.

Let $\left\{\omega^{I}\right\}$ be a basis of the space of polynomials in the $C_{\mu \nu}^{a}, \widehat{T}_{\alpha \mu \mid \nu}^{a}$ and $\widehat{U}_{\alpha \mu \mid \beta \nu}^{a}$ (since these variables anticommute, this space is finite-dimensional). If a local form $a$ is $\gamma$-closed, we have

$$
\gamma a=0 \Rightarrow a=\alpha_{J}\left(\left[\Phi^{i *}\right],[K],\{F\}\right) \omega^{J}\left(C_{\mu \nu}^{a}, \widehat{T}_{\alpha \mu \mid \nu}^{a}, \widehat{U}_{\alpha \mu \mid \beta \nu}^{a}\right)+\gamma b
$$

If $a$ has a fixed, finite ghost number, then $a$ can only contain a finite number of antifields. Moreover, since the local form a possesses a finite number of derivatives, we find that the $\alpha_{J}$ are polynomials. Such a polynomial $\alpha_{J}\left(\left[\Phi^{i *}\right],[K],\{F\}\right)$ will be called an invariant polynomial.

Remark 1: Because of the Damour-Deser identity [28]

$$
\eta^{\alpha \beta} K_{\alpha \mu|\beta \nu| \gamma \rho} \equiv 2 \partial_{[\gamma} F_{\rho] \mu \nu},
$$


the derivatives of the Fronsdal tensor are not all independent of the curvature tensor $K$. This is why, in Proposition 1, the completely symmetrized derivatives of $F$ appear, together with all the derivatives of the curvature $K$. However, from now on, we will assume that every time the trace $\eta^{\alpha \beta} K_{\alpha \mu|\beta \nu| \gamma \rho}$ appears, we substitute $2 \partial_{[\gamma} F_{\rho] \mu \nu}$ for it. With this convention, we can write $\alpha_{J}\left(\left[\Phi^{i *}\right],[K],[F]\right)$ instead of the unconvenient notation $\alpha_{J}\left(\left[\Phi^{i *}\right],[K],\{F\}\right)$.

Remark 2: It is possible to make a link with the variables occurring in the frame-like first-order formulation of free massless spin-3 field in Minkowski space-time [32]. There, the spin-3 field is represented off-shell by a frame-like object $e_{\mu \mid a b}$, symmetric and traceless in the internal indices $(a, b)$. The spin-3 connection $\omega_{\mu|b| a_{1} a_{2}}$ is traceless in the internal Latin indices, symmetric in $\left(a_{1}, a_{2}\right)$ and obeying $\omega_{\mu \mid\left(b \mid a_{1} a_{2}\right)} \equiv 0$. The gauge transformations are $\delta e_{\mu \mid a b}=\partial_{\mu} \xi_{a b}+\alpha_{\mu \mid a b}, \delta \omega_{\mu|b| a_{1} a_{2}}=\partial_{\mu} \alpha_{b \mid a_{1} a_{2}}+\Sigma_{\mu|b| a_{1} a_{2}}$, where the parameter $\xi_{a b}$ is symmetric and traceless in $(a, b)$, the generalized Lorentz parameter $\alpha_{\mu \mid a b}$ is completely traceless, symmetric in $(a, b)$ and satisfies the identity $\alpha_{(\mu \mid a b)} \equiv 0$. Finally, the parameter $\Sigma_{\mu|a| b c}$ transforms in the $o(n-1,1)$ irreducible representation associated with the Young tableau \begin{tabular}{|l|l|}
$\mu$ & $a$ \\
\hline & $c$
\end{tabular} , in the manifestly symmetric convention. By choosing the generalized Lorentz parameter appropriately, it is possible to work in the gauge where the frame-field $e_{\mu \mid a b}$ is completely symmetric, $e_{\mu \mid a b}=e_{(\mu \mid a b)} \equiv h_{\mu a b}$. Then, it is still possible to perform a gauge transformation with parameters $\alpha_{\mu \mid a b}$ and $\xi_{a b}$, provided the traceless component of $\partial_{[\mu} \xi_{a] b}$ be equal to $-\alpha_{[\mu \mid a] b}$. The traceless component of $\partial_{[\mu} \xi_{a] b}$ is nothing but the variable $\widehat{T}_{\mu \alpha \mid \beta}$ in the BRST conventions. Furthermore, in the 1.5 formalism where the connection is still present in the action, but viewed as a function of $e_{\mu \mid a_{1} a_{2}}$, consistency with the "symmetric gauge" $e_{\mu \mid a b}=e_{(\mu \mid a b)} \equiv h_{\mu a b}$ implies that the traceless component of the second derivative $\partial_{[a} \xi_{b][c, \mu]}$ be entirely determined by $\Sigma_{\mu|b| a c}$. The traceless component of $\partial_{[a} \xi_{b][c, \mu]}$ is the variable $\widehat{U}_{\alpha \beta \mid \gamma \mu}$ in the BRST language. The relations $\widehat{T}_{\mu \alpha \mid \beta} \longleftrightarrow \alpha_{\mu \mid a b}$ and $\widehat{U}_{\alpha \beta \mid \gamma \mu} \longleftrightarrow \Sigma_{\mu|b| a c}$ are now manifest (note the we work in the manifestly antisymmetric convention, as opposed to the choice made in [32]). The variables $\left\{C_{\mu \nu}, \widehat{T}_{\mu \alpha \mid \beta}, \widehat{U}_{\alpha \beta \mid \gamma \mu}\right\} \in H(\gamma)$ in the ghost sector are in one-to-one correspondence with the gauge parameters $\left\{\xi_{\mu \nu}, \alpha_{\mu \mid a b}, \Sigma_{\mu|b| a c}\right\}$ of the first-order formalism [32].

\subsection{Invariant Poincaré lemma}

We shall need several standard results on the cohomology of $d$ in the space of invariant polynomials.

Proposition 2. In form degree less than $n$ and in antifield number strictly greater than 0 , the cohomology of $d$ is trivial in the space of invariant polynomials. That is to say, if $\alpha$ is an invariant polynomial, the equation $d \alpha=0$ with antigh $(\alpha)>0$ implies $\alpha=d \beta$ where $\beta$ is also an invariant polynomial. 
The latter property is rather generic for gauge theories (see e.g. Ref. [22] for a proof), as well as the following:

Proposition 3. If a has strictly positive antifield number, then the equation $\gamma a+d b=0$ is equivalent, up to trivial redefinitions, to $\gamma a=0$. More precisely, one can always add d-exact terms to $a$ and get a cocycle $a^{\prime}:=a+d c$ of $\gamma$, such that $\gamma a^{\prime}=0$.

Proof: Along the lines of Ref. [22], we consider the descent associated with $\gamma a+d b=0$ : from this equation, one infers, by using the properties $\gamma^{2}=0, \gamma d+d \gamma=0$ and the triviality of the cohomology of $d$, that $\gamma b+d c=0$ for some $c$. Going on in the same way, we build a "descent"

$$
\begin{aligned}
\gamma a+d b & =0 \\
\gamma b+d c & =0 \\
\gamma c+d e & =0 \\
& \vdots \\
\gamma m+d n & =0 \\
\gamma n & =0 .
\end{aligned}
$$

in which each successive equation has one less unit of form-degree. The descent ends with $\gamma n=0$ either because $n$ is a zero-form, or because one stops earlier with a $\gamma$-closed term. Now, because $n$ is $\gamma$-closed, one has, up to trivial, irrelevant terms, $n=\alpha_{J} \omega^{J}$. Inserting this into the previous equation in the descent yields

$$
d\left(\alpha_{J}\right) \omega^{J} \pm \alpha_{J} d \omega^{J}+\gamma m=0 .
$$

In order to analyse this equation, we introduce a new differential.

Definition (differential $D$ ): The action of the differential $D$ on $h_{\mu \nu \rho}^{a}, h_{a}^{* \mu \nu \rho}, C_{a}^{* \mu \nu}$ and all their derivatives is the same as the action of the total derivative $d$, but its action on the ghosts is given by :

$$
\begin{aligned}
D C_{\mu \nu}^{a} & =\frac{4}{3} d x^{\alpha} \widehat{T}_{\alpha(\mu \mid \nu)}^{a}, \\
D \widehat{T}_{\mu \alpha \mid \beta}^{a} & =d x^{\rho} \widehat{U}_{\mu \alpha \mid \rho \beta}^{a}, \\
D\left(\partial_{\rho_{1} \ldots \rho_{t}} C_{\mu}\right) & =0 \text { if } t \geqslant 2 .
\end{aligned}
$$

The above definitions follow from

$$
\begin{aligned}
\partial_{\alpha} C_{\mu \nu}^{a} & =\frac{1}{3}\left(\gamma h_{\alpha \mu \nu}^{a}\right)+\frac{4}{3} T_{\alpha(\mu \mid \nu),}^{a} \\
\partial_{\rho} T_{\mu \alpha \mid \beta} & =-\frac{1}{2} \gamma\left(\partial_{[\alpha} h_{\mu] \beta \rho}\right)+U_{\mu \alpha \mid \rho \beta}, \\
\partial_{\rho} U_{\mu \alpha \mid \nu \beta} & =\frac{1}{3} \gamma\left(\partial_{[\mu} h_{\alpha] \rho[\beta, \nu]}\right) .
\end{aligned}
$$


The operator $D$ thus coincides with $d$ up to $\gamma$-exact terms.

It follows from the definitions that $D \omega^{J}=A^{J}{ }_{I} \omega^{I}$ for some constant matrix $A^{J}$ that involves $d x^{\mu}$ only. One can rewrite (4.20) as

$$
\underbrace{d\left(\alpha_{J}\right) \omega^{J} \pm \alpha_{J} D \omega^{J}}_{=\left(d \alpha_{J} \pm \alpha_{I} A^{I} J\right) \omega^{J}}+\gamma m^{\prime}=0
$$

which implies,

$$
d\left(\alpha_{J}\right) \omega^{J} \pm \alpha_{J} D \omega^{J}=0
$$

since a term of the form $\beta_{J} \omega^{J}$ (with $\beta_{J}$ invariant) is $\gamma$-exact if and only if it is zero. It is also convenient to introduce a new grading.

Definition (D-degree): The number of $\widehat{T}_{\alpha \mu \mid \nu}$ 's plus two times the number of $\widehat{U}_{\alpha \mu \mid \beta \nu}$ 's is called the $D$-degree. It is bounded because there is a finite number of $\widehat{T}_{\alpha \mu \mid \nu}$ 's and $\widehat{U}_{\alpha \mu \mid \beta \nu}$ 's, which are anticommuting. The operator $D$ splits as the sum of an operator $D_{1}$ that raises the $D$-degree by one unit, and an operator $D_{0}$ that leaves it unchanged. $D_{0}$ has the same action as $d$ on $h_{\mu \nu \rho}, h^{* \mu \nu \rho}, C^{* \alpha \beta}$ and all their derivatives, and gives 0 when acting on the ghosts. $D_{1}$ gives 0 when acting on all the variables but the ghosts on which it reproduces the action of $D$.

Let us expand (4.20) according to the $D$-degree. At lowest order, we get

$$
d \alpha_{J_{0}}=0
$$

where $J_{0}$ labels the $\omega^{J}$ that contain no derivative of the ghosts $\left(D \omega^{J}=D_{1} \omega^{J}\right.$ contains at least one derivative). This equation implies, according to Proposition 2 , that $\alpha_{J_{0}}=d \beta_{J_{0}}$ where $\beta_{J_{0}}$ is an invariant polynomial. Accordingly, one can write

$$
\alpha_{J_{0}} \omega^{J_{0}}=d\left(\beta_{J_{0}} \omega^{J_{0}}\right) \mp \beta_{J_{0}} D \omega^{J_{0}}+\gamma \text {-exact terms. }
$$

The term $\beta_{J_{0}} D \omega^{J_{0}}$ has $D$-degree equal to 1 . Thus, by adding trivial terms to the last term $n\left(=\alpha_{J} \omega^{J}\right)$ in the descent (4.19), we can assume that it does not contain any term of $D$ degree 0 . One can then successively remove the terms of $D$-degree $1, D$-degree 2 , etc, until one gets $n=0$. One then repeats the argument for $m$ and the previous terms in the descent (4.19) until one gets $b=0$, i.e., $\gamma a=0$, as requested.

\subsection{Cohomology of $\delta$ modulo $d: H_{k}^{n}(\delta \mid d)$}

In this section, we review the local Koszul-Tate cohomology groups in top form-degree and antighost numbers $k \geqslant 2$. The group $H_{1}^{D}(\delta \mid d)$ describes the infinitely many conserved currents and will not be studied here.

Let us first recall a general theorem (Theorem 9.1 in [33]). 
Proposition 4. For a linear gauge theory of reducibility order $r$,

$$
H_{p}^{n}(\delta \mid d)=0 \text { for } p>r+2 .
$$

Since the theory at hand has no reducibility, we are left with the computation of $H_{2}^{n}(\delta \mid d)$. The cohomology $H_{2}^{n}(\delta \mid d)$ is given by the following theorem.

Proposition 5. A complete set of representatives of $H_{2}^{n}(\delta \mid d)$ is given by the antifields $C_{a}^{* \mu \nu}$, up to explicitly $x$-dependent terms. In detail,

$$
\left.\begin{array}{c}
\delta a_{2}^{n}+d b_{1}^{n-1}=0, \\
a_{2}^{n} \sim a_{2}^{n}+\delta c_{3}^{n}+d c_{2}^{n-1}
\end{array}\right\} \Longleftrightarrow\left\{\begin{array}{l}
a_{2}^{n}=L_{\mu \nu}^{a}(x) C_{a}^{* \mu \nu} d^{n} x+\delta b_{3}^{n}+d b_{2}^{n-1}, \\
L_{\mu \nu}^{a}(x)=\lambda_{\mu \nu}^{a}+A_{\mu \nu \mid \rho}^{a} x^{\rho}+B_{\mu \nu \mid \rho \sigma}^{a} x^{\rho} x^{\sigma} .
\end{array}\right.
$$

The constant tensor $\lambda_{\mu \nu}^{a}$ is symmetric and traceless in the indices $\mu \nu$, and so are the constant tensors $A_{\mu \nu \mid \rho}^{a}$ and $B_{\mu \nu \mid \rho \sigma}^{a}$. Moreover, the tensors $A_{\mu \nu \mid \rho}^{a}$ and $B_{\mu \nu \mid \rho \sigma}^{a}$ transform in the irreducible representations of $G L(n, \mathbb{R})$ labeled by the Young tableaux $\frac{\mu \nu \nu}{\rho}$ and $\frac{\mu \nu \nu}{\rho \mid \sigma}$, meaning that

$$
\begin{aligned}
A_{\mu \nu \mid \rho}^{a} & =A_{\nu \mu \mid \rho}^{a}, \quad A_{(\mu \nu \mid \rho)}^{a} \equiv 0, \\
B_{\mu \nu \mid \rho \sigma}^{a} & =B_{\nu \mu \mid \rho \sigma}^{a}=B_{\mu \nu \mid \sigma \rho}^{a}, \quad B_{(\mu \nu \mid \rho) \sigma}^{a}=0 .
\end{aligned}
$$

Together with the tracelessness constraints on the constant tensors $A_{\mu \nu \mid \rho}^{a}$ and $B_{\mu \nu \mid \rho \sigma}^{a}$, the $G l(n, \mathbb{R})$ irreducibility conditions written here above imply that the tensors $\lambda_{\mu \nu}^{a}, A_{\mu \nu \mid \rho}^{a}$ and $B_{\mu \nu \mid \rho \sigma}^{a}$ respectively transform in the irreducible representations of $O(n-1,1)$ labeled by the Young tableaux $\mu \nu, \frac{\mu \nu}{\rho}$ and $\frac{\mu \nu \nu}{\rho \mid \sigma}$.

The proof of Proposition 5 in the general spin- $s$ case has been given in Ref. [24] (see also [34]). The spin-3 case under consideration was already written in Ref. [35].

\subsection{Invariant cohomology of $\delta$ modulo $d: H_{k}^{n, i n v}(\delta \mid d)$}

We have studied above the cohomology of $\delta$ modulo $d$ in the space of arbitary local functions of the fields $h_{\mu \nu \rho}^{a}$, the antifields $\Phi^{* i}$, and their derivatives. One can also study $H_{k}^{n}(\delta \mid d)$ in the space of invariant polynomials in these variables, which involve $h_{\mu \nu \rho}^{a}$ and its derivatives only through the curvature $K$, the Fronsdal tensor $F$, and their derivatives (as well as the antifields and their derivatives). The above theorems remain unchanged in this space, i.e. $H_{k}^{n, \text { inv }}(\delta \mid d) \cong 0$ for $k>2$. This very nontrivial property is crucial for the computation of $H^{n, 0}(s \mid d)$ and is a consequence of

Theorem 3. Assume that the invariant polynomial $a_{k}^{p}(p=$ form-degree, $k=$ antifield number) is $\delta$-trivial modulo $d$,

$$
a_{k}^{p}=\delta \mu_{k+1}^{p}+d \mu_{k}^{p-1} \quad(k \geqslant 2) .
$$

Then, one can always choose $\mu_{k+1}^{p}$ and $\mu_{k}^{p-1}$ to be invariant. 
To prove the theorem, we need the following lemma, a proof of which can be found e.g. in $[22]$.

Lemma 2. If $a$ is an invariant polynomial that is $\delta$-exact, $a=\delta b$, then, a is $\delta$-exact in the space of invariant polynomials. That is, one can take $b$ to be also invariant.

The next two subsections are devoted to the proof of Theorem 3.

\subsubsection{Propagation of the invariance in form degree}

We first derive a chain of equations with the same structure as (4.28) [36]. Acting with $d$ on (4.28), we get $d a_{k}^{p}=-\delta d \mu_{k+1}^{p}$. Using the lemma and the fact that $d a_{k}^{p}$ is invariant, we can also write $d a_{k}^{p}=-\delta a_{k+1}^{p+1}$ with $a_{k+1}^{p+1}$ invariant. Substituting this into $d a_{k}^{p}=-\delta d \mu_{k+1}^{p}$, we get $\delta\left[a_{k+1}^{p+1}-d \mu_{k+1}^{p}\right]=0$. As $H(\delta)$ is trivial in antifield number $>0$, this yields

$$
a_{k+1}^{p+1}=\delta \mu_{k+2}^{p+1}+d \mu_{k+1}^{p}
$$

which has the same structure as (4.28). We can then repeat the same operations, until we reach form-degree $n$,

$$
a_{k+n-p}^{n}=\delta \mu_{k+n-p+1}^{n}+d \mu_{k+n-p}^{n-1} .
$$

Similarly, one can go down in form-degree. Acting with $\delta$ on (4.28), one gets $\delta a_{k}^{p}=$ $-d\left(\delta \mu_{k}^{p-1}\right)$. If the antifield number $k-1$ of $\delta a_{k}^{p}$ is greater than or equal to one (i.e., $k>1$ ), one can rewrite, thanks to Proposition $2, \delta a_{k}^{p}=-d a_{k-1}^{p-1}$ where $a_{k-1}^{p-1}$ is invariant. (If $k=1$ we cannot go down and the bottom of the chain is (4.28) with $k=1$, namely $a_{1}^{p}=\delta \mu_{2}^{p}+d \mu_{1}^{p-1}$.) Consequently $d\left[a_{k-1}^{p-1}-\delta \mu_{k}^{p-1}\right]=0$ and, as before, we deduce another equation similar to (4.28) :

$$
a_{k-1}^{p-1}=\delta \mu_{k}^{p-1}+d \mu_{k-1}^{p-1} .
$$

Applying $\delta$ on this equation the descent continues. This descent stops at form degree zero or antifield number one, whichever is reached first, i.e.,

$$
\begin{array}{cl}
\text { either } & a_{k-p}^{0}=\delta \mu_{k-p+1}^{0} \\
\text { or } & a_{1}^{p-k+1}=\delta \mu_{2}^{p-k+1}+d \mu_{1}^{p-k} .
\end{array}
$$

Putting all these observations together we can write the entire descent as

$$
\begin{aligned}
a_{k+n-p}^{n} & =\delta \mu_{k+n-p+1}^{n}+d \mu_{k+n-p}^{n-1} \\
& \vdots \\
a_{k+1}^{p+1} & =\delta \mu_{k+2}^{p+1}+d \mu_{k+1}^{p} \\
a_{k}^{p} & =\delta \mu_{k+1}^{p}+d \mu_{k}^{p-1}
\end{aligned}
$$




$$
\begin{aligned}
\qquad a_{k-1}^{p-1} & =\delta \mu_{k}^{p-1}+d \mu_{k-1}^{p-2} \\
& \vdots \\
\text { either } a_{k-p}^{0} & =\delta \mu_{k-p+1}^{0} \\
\text { or } a_{1}^{p-k+1} & =\delta \mu_{2}^{p-k+1}+d \mu_{1}^{p-k}
\end{aligned}
$$

where all the $a_{k \pm i}^{p \pm i}$ are invariants.

Let us show that when one of the $\mu$ 's in the chain is invariant, we can actually choose all the other $\mu$ 's in such a way that they share this property. In other words, the invariance

property propagates up and down in the ladder. Let us thus assume that $\mu_{b}^{c-1}$ is invariant. This $\mu_{b}^{c-1}$ appears in two equations of the descent :

$$
\begin{aligned}
a_{b}^{c} & =\delta \mu_{b+1}^{c}+d \mu_{b}^{c-1}, \\
a_{b-1}^{c-1} & =\delta \mu_{b}^{c-1}+d \mu_{b-1}^{c-2}
\end{aligned}
$$

(if we are at the bottom or at the top, $\mu_{b}^{c-1}$ occurs in only one equation, and one should just proceed from that one). The first equation tells us that $\delta \mu_{b+1}^{c}$ is invariant. Thanks to Lemma 2 we can choose $\mu_{b+1}^{c}$ to be invariant. Looking at the second equation, we see that $d \mu_{b-1}^{c-2}$ is invariant and by virtue of Proposition $2, \mu_{b-1}^{c-2}$ can be chosen to be invariant since the antifield number $b$ is positive. These two $\mu$ 's appear each one in two different equations of the chain, where we can apply the same reasoning. The invariance property propagates then to all the $\mu$ 's. Consequently, it is enough to prove the theorem in form degree $n$.

\subsubsection{Top form degree}

Two cases may be distinguished depending on whether the antifield number $k$ is greater than $n$ or not.

In the first case, one can prove the following lemma:

Lemma 3. If $a_{k}^{n}$ is of antifield number $k>n$, then the " $\mu$ " $s$ in (4.28) can be taken to be invariant.

Proof for $k>n$ : If $k>n$, the last equation of the descent is $a_{k-n}^{0}=\delta \mu_{k-n+1}^{0}$. We can, using Lemma 2 , choose $\mu_{k-n+1}^{0}$ invariant, and so, all the $\mu$ 's can be chosen to have the same property.

It remains therefore to prove Theorem 3 in the case where the antifield number satisfies $k \leqslant n$. Rewriting the top equation (i.e. (4.28) with $p=n$ ) in dual notation, we have

$$
a_{k}=\delta b_{k+1}+\partial_{\rho} j_{k}^{\rho},(k \geqslant 2) .
$$


We will work by induction on the antifield number, showing that if the property expressed in Theorem 3 is true for $k+1$ (with $k>1$ ), then it is true for $k$. As we already know that it is true in the case $k>n$, the theorem will be proved.

Inductive proof for $k \leqslant n$ : The proof follows the lines of Ref. [36] and decomposes in two parts. First, all Euler-Lagrange derivatives of (4.35) are computed. Second, the EulerLagrange (E.L.) derivative of an invariant quantity is also invariant. This property is used to express the E.L. derivatives of $a_{k}$ in terms of invariants only. Third, the homotopy formula is used to reconstruct $a_{k}$ from its E.L. derivatives. This almost ends the proof.

(i) Let us take the E.L. derivatives of (4.35). Since the E.L. derivatives with respect to the $C_{\alpha}^{*}$ commute with $\delta$, we get first :

$$
\frac{\delta^{L} a_{k}}{\delta C_{\alpha \beta}^{*}}=\delta Z_{k-1}^{\alpha \beta}
$$

with $Z_{k-1}^{\alpha \beta}=\frac{\delta^{L} b_{k+1}}{\delta C_{\alpha \beta}^{*}}$. For the E.L. derivatives of $b_{k+1}$ with respect to $h_{\mu \nu \rho}^{*}$ we obtain, after a direct computation,

$$
\frac{\delta^{L} a_{k}}{\delta h_{\mu \nu \rho}^{*}}=-\delta X_{k}^{\mu \nu \rho}+3 \partial^{(\mu} Z_{k-1}^{\nu \rho)}
$$

where $X_{k}^{\mu \nu \rho}=\frac{\delta^{L} b_{k+1}}{\delta h_{\mu \nu \rho}^{*}}$. Finally, let us compute the E.L. derivatives of $a_{k}$ with respect to the fields. We get :

$$
\frac{\delta^{L} a_{k}}{\delta h_{\mu \nu \rho}}=\delta Y_{k+1}^{\mu \nu \rho}+\mathcal{G}^{\mu \nu \rho \mid \alpha \beta \gamma} X_{\alpha \beta \gamma \mid k}
$$

where $Y_{k+1}^{\mu \nu \rho}=\frac{\delta^{L} b_{k+1}}{\delta h_{\mu \nu \rho}}$ and $\mathcal{G}^{\mu \nu \rho \mid \alpha \beta \gamma}(\partial)$ is the second-order self-adjoint differential operator appearing in the equations of motion (2.3):

$$
G^{\mu \nu \rho}=\mathcal{G}^{\mu \nu \rho \mid \alpha \beta \gamma} h_{\alpha \beta \gamma}
$$

The hermiticity of $\mathcal{G}$ implies $\mathcal{G}^{\mu \nu \rho \mid \alpha \beta \gamma}=\mathcal{G}^{\alpha \beta \gamma \mid \mu \nu \rho}$.

(ii) The E.L. derivatives of an invariant object are invariant. Thus, $\frac{\delta^{L} a_{k}}{\delta C_{\alpha \beta}^{*}}$ is invariant. Therefore, by Lemma 2 and Eq. (4.36), we have also

$$
\frac{\delta^{L} a_{k}}{\delta C_{\alpha \beta}^{*}}=\delta Z_{k-1}^{\alpha \beta}
$$

for some invariant $Z_{k-1}^{\alpha \beta}$. Indeed, let us write the decomposition $Z_{k-1}^{\alpha \beta}=Z_{k-1}^{\prime \alpha \beta}+\tilde{Z}_{k-1}^{\alpha \beta}$, where $\tilde{Z}_{k-1}^{\alpha \beta}$ is obtained from $Z_{k-1}^{\alpha \beta}$ by setting to zero all the terms that belong only to $H(\gamma)$. The 
latter operation clearly commutes with taking the $\delta$ of something, so that Eq. (4.36) gives $0=\delta \tilde{Z}_{k-1}^{\alpha \beta}$ which, by the acyclicity of $\delta$, yields $\tilde{Z}_{k-1}^{\alpha \beta}=\delta \sigma_{k}^{\alpha \beta}$ where $\sigma_{k}^{\alpha \beta}$ can be chosen to be traceless. Substituting $\delta \sigma_{k}^{\alpha \beta}+Z_{k-1}^{\alpha \beta}$ for $Z_{k-1}^{\alpha \beta}$ in Eq. (4.36) gives Eq. (4.39).

Similarly, one easily verifies that

$$
\frac{\delta^{L} a_{k}}{\delta h_{\mu \nu \rho}^{*}}=-\delta X_{k}^{\prime \mu \nu \rho}+3 \partial^{(\mu} Z_{k-1}^{\prime \nu \rho)},
$$

where $X_{k}^{\mu \nu \rho}=X_{k}^{\prime \mu \nu \rho}+3 \partial^{(\mu} \sigma_{k}^{\nu \rho)}+\delta \rho_{k+1}^{\mu \nu \rho}$. Finally, using $\mathcal{G}^{\mu \nu \rho}{ }_{\alpha \beta \gamma} \partial^{(\alpha} \sigma^{\beta \gamma)}{ }_{k}=0$ due to the gauge invariance of the equations of motion ( $\sigma_{\alpha \beta}$ has been taken traceless), we find

$$
\frac{\delta^{L} a_{k}}{\delta h_{\mu \nu \rho}}=\delta Y_{k+1}^{\prime \mu \nu \rho}+\mathcal{G}_{\alpha \beta \gamma}^{\mu \nu \rho} X_{k}^{\prime \beta \gamma}
$$

for the invariants $X_{k}^{\prime \mu \nu \rho}$ and $Y_{k+1}^{\prime \mu \nu \rho}$. Before ending the argument by making use of the homotopy formula, it is necessary to know more about the invariant $Y_{k+1}^{\mu \nu \rho}$.

Since $a_{k}$ is invariant, it depends on the fields only through the curvature $K$, the Fronsdal tensor and their derivatives. (We remind the reader of our convention of Section 4.3 to substitute $2 \partial_{[\gamma} F_{\rho] \mu \nu}$ for $\eta^{\alpha \beta} K_{\alpha \mu|\beta \nu| \gamma \rho}$ everywhere.) We then express the Fronsdal tensor in terms of the Einstein tensor (2.4): $F_{\mu \nu \rho}=G_{\mu \nu \rho}-\frac{3}{n} \eta_{(\mu \nu} G_{\rho)}$, so that we can write $a_{k}=$ $a_{k}\left(\left[\Phi^{* i}\right],[K],[G]\right)$, where $[G]$ denotes the Einstein tensor and its derivatives. We can thus write

$$
\frac{\delta^{L} a_{k}}{\delta h_{\mu \nu \rho}}=\mathcal{G}^{\mu \nu \rho}{ }_{\alpha \beta \gamma} A_{k}^{\prime \alpha \beta \gamma}+\partial_{\alpha} \partial_{\beta} \partial_{\gamma} M_{k}^{\prime \alpha \mu|\beta \nu| \gamma \rho}
$$

where

$$
A_{k}^{\prime \alpha \beta \gamma} \propto \frac{\delta a_{k}}{\delta G_{\alpha \beta \gamma}}
$$

and

$$
M_{k}^{\prime \alpha \mu|\beta \nu| \gamma \rho} \propto \frac{\delta a_{k}}{\delta K_{\alpha \mu|\beta \nu| \gamma \rho}}
$$

are both invariant and respectively have the same symmetry properties as the "Einstein" and "Riemann" tensors.

Combining Eq. (4.41) with Eq. (4.42) gives

$$
\delta Y_{k+1}^{\prime \mu \nu \rho}=\partial_{\alpha} \partial_{\beta} \partial_{\gamma} M_{k}^{\prime \alpha \mu|\beta \nu| \gamma \rho}+\mathcal{G}^{\mu \nu \rho}{ }_{\alpha \beta \gamma} B_{k}^{\prime \alpha \beta \gamma}
$$

with ${B^{\prime}}_{k}^{\alpha \beta \gamma}:=A_{k}^{\prime \alpha \beta \gamma}-{X^{\prime}}_{k}^{\alpha \beta \gamma}$. Now, only the first term on the right-hand-side of Eq. (4.43) is divergence-free, $\partial_{\mu}\left(\partial_{\alpha \beta \gamma} M_{k}^{\prime \alpha \mu|\beta \nu| \gamma \rho}\right) \equiv 0$, not the second one which instead obeys a relation analogous to the Noether identities (2.6). As a result, we have $\delta\left[\partial_{\mu}\left(Y_{k+1}^{\prime \mu \nu \rho}-\frac{1}{n} \eta^{\nu \rho} Y_{k+1}^{\prime \mu}\right)\right]=0$, where $Y_{k+1}^{\prime \mu} \equiv \eta_{\nu \rho} Y_{k+1}^{\mu \nu \rho}$. By Lemma 2, we deduce

$$
\partial_{\mu}\left(Y_{k+1}^{\mu \nu \rho}-\frac{1}{n} \eta^{\nu \rho} Y_{k+1}^{\prime \mu}\right)+\delta F_{k+2}^{\prime \nu \rho}=0
$$


where $F_{k+2}^{\prime \nu \rho}$ is invariant and can be chosen symmetric and traceless. Eq. (4.44) determines a cocycle of $H_{k+1}^{n-1}(d \mid \delta)$, for given $\nu$ and $\rho$. Using the general isomorphisms $H_{k+1}^{n-1}(d \mid \delta) \cong$ $H_{k+2}^{n}(\delta \mid d) \cong 0(k \geqslant 1)[33]$ gives

$$
Y_{k+1}^{\prime \mu \nu \rho}-\frac{1}{n} \eta^{\nu \rho} Y_{k+1}^{\prime \mu}=\partial_{\alpha} T_{k+1}^{\alpha \mu \mid \nu \rho}+\delta P_{k+2}^{\mu \nu \rho},
$$

where both $T_{k+1}^{\alpha \mu \mid \nu \rho}$ and $P_{k+2}^{\mu \nu \rho}$ are invariant by the induction hypothesis. Moreover, $T_{k+1}^{\alpha \mu \mid \nu \rho}$ is antisymmetric in its first two indices. The tensors $T_{k+1}^{\alpha \mu \mid \nu \rho}$ and $P_{k+2}^{\mu \nu \rho}$ are both symmetrictraceless in $(\nu, \rho)$. This results easily from taking the trace of Eq. (4.45) with $\eta_{\nu \rho}$ and using the general isomorphisms $H_{k+1}^{n-2}(d \mid \delta) \cong H_{k+2}^{n-1}(\delta \mid d) \cong H_{k+3}^{n}(\delta \mid d) \cong 0$ [33] which hold since $k$ is positive. From Eq. (4.45) we obtain

$$
Y_{k+1}^{\prime \mu \nu \rho}=\partial_{\alpha}\left[T_{k+1}^{\alpha \mu \mid \nu \rho}+\frac{1}{n-1} \eta^{\nu \rho} T_{k+1}^{\alpha \mid \mu}\right]+\delta\left[P_{k+2}^{\mu \nu \rho}+\frac{1}{n-1} \eta^{\nu \rho} P_{k+2}^{\mu}\right]
$$

where $T_{k+1}^{\alpha \mid \mu} \equiv \eta_{\nu \rho} T_{k+1}^{\alpha \nu \mid \rho \mu}$ and $P_{k+2}^{\mu} \equiv \eta_{\nu \rho} P_{k+2}^{\nu \rho \mu}$. Since $Y_{k+1}^{\prime \mu \nu \rho}$ is symmetric in $\mu$ and $\nu$, we have also $\partial_{\alpha}\left[T_{k+1}^{\alpha[\mu \mid \nu] \rho}+\frac{1}{n-1} T_{k+1}^{\alpha \mid[\mu} \eta^{\nu] \rho}\right]+\delta\left[P_{k+2}^{[\mu \nu] \rho}+\frac{1}{n-1} \eta^{\rho[\nu} P_{k+2}^{\mu]}\right]=0$. The triviality of $H_{k+2}^{n}(d \mid \delta)$ $(k>0)$ implies again that $\left(P_{k+2}^{[\mu \nu] \rho}+\frac{1}{n-1} \eta^{\rho[\nu} P_{k+2}^{\mu]}\right)$ and $\left(T_{k+1}^{\alpha[\mu \mid \nu] \rho}+\frac{1}{n-1} T_{k+1}^{\alpha \mid[\mu} \eta^{\nu] \rho}\right)$ are trivial, in particular,

$$
T_{k+1}^{\alpha[\mu \mid \nu] \rho}+\frac{1}{n-1} T_{k+1}^{\alpha \mid[\mu} \eta^{\nu] \rho}=\partial_{\beta} S_{k+1}^{\beta \alpha|\mu \nu| \rho}+\delta Q_{k+2}^{\alpha \mu \nu \rho}
$$

where $S_{k+1}^{\beta \alpha|\mu \nu| \rho}$ is antisymmetric in $(\beta, \alpha)$ and $(\mu, \nu)$. Moreover, it is traceless in $\mu, \nu, \rho$ as the left hand side of the above equation shows. The induction assumption allows us to choose $S_{k+1}^{\beta \alpha|\mu \nu| \rho}$ and $Q_{k+2}^{\alpha \mu \nu \rho}$ invariant. We now project both sides of Eq. (4.47) on the symmetries of the Weyl tensor. For example, denoting by $W_{k+1}^{\beta|\mu \nu| \alpha \rho}$ the projection $\mathcal{W}_{\mu^{\prime} \nu^{\prime} \alpha^{\prime} \rho^{\prime}}^{\mu \nu \alpha} S_{k+1}^{\beta \alpha^{\prime}\left|\mu^{\prime} \nu^{\prime}\right| \rho^{\prime}}$ of $S_{k+1}^{\beta \alpha|\mu \nu| \rho}$, we have

$$
\begin{aligned}
W_{k+1}^{\beta|\mu \nu| \alpha \rho} & =W_{k+1}^{\beta|\alpha \rho| \mu \nu}=-W_{k+1}^{\beta|\nu \mu| \alpha \rho}=-W_{k+1}^{\beta|\mu \nu| \rho \alpha} \\
W_{k+1}^{\beta \mid \mu[\nu \mid \alpha \rho]} & =0, \quad \eta_{\mu \alpha} W_{k+1}^{\beta|\mu \nu| \alpha \rho}=0 .
\end{aligned}
$$

As a consequence of the symmetries of $T_{k+1}^{\alpha \mu \mid \nu \rho}$, the projection of Eq. (4.47) on the symmetries of the Weyl tensor gives

$$
0=\partial_{\beta} W_{k+1}^{\beta|\mu \nu| \alpha \rho}+\delta(\ldots)
$$

where we do not write the (invariant) $\delta$-exact terms explicitly because they play no role in what follows. Eq. (4.48) determines, for given $(\mu, \nu, \alpha, \rho)$, a cocycle of $H_{k+1}^{n-1}(d \mid \delta, H(\gamma))$. Using again the isomorphisms [33] $H_{k+1}^{n-1}(d \mid \delta) \cong H_{k+2}^{n}(\delta \mid d) \cong 0(k \geqslant 1)$ and the induction hypothesis, we find

$$
W_{k+1}^{\beta|\mu \nu| \alpha \rho}=\partial_{\gamma} \phi_{k+1}^{\gamma \beta|\mu \nu| \alpha \rho}+\delta(\ldots)
$$


where $\phi_{k+1}^{\gamma \beta|\mu \nu| \alpha \rho}$ is invariant, antisymmetric in $(\gamma, \beta)$ and possesses the symmetries of the Weyl tensor in its last four indices. The $\delta$-exact term is invariant as well. Then, projecting the invariant tensor $4 \phi_{k+1}^{\gamma \beta|\mu \nu| \alpha \rho}$ on the symmetries of the curvature tensor $K^{\gamma \beta|\mu \nu| \alpha \rho}$ and calling the result $\Psi_{k+1}^{\gamma \beta|\mu \nu| \alpha \rho}$ which is of course invariant, we find after some rather lengthy algebra (which takes no time using Ricci [37])

$$
Y_{k+1}^{\prime \mu \nu \rho}=\partial_{\alpha} \partial_{\beta} \partial_{\gamma} \Psi_{k+1}^{\alpha \mu|\beta \nu| \gamma \rho}+\mathcal{G}^{\mu \nu \rho}{ }_{\alpha \beta \gamma} \widehat{X}_{k+1}^{\alpha \beta \gamma}+\delta(\ldots)
$$

with

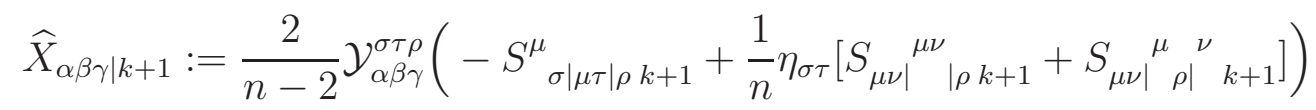

where $\mathcal{Y}_{\alpha \beta \gamma}^{\sigma \tau \rho}=\mathcal{Y}_{(\alpha \beta \gamma)}^{(\sigma \tau \rho)}$ projects on completely symmetric rank-3 tensors.

(iii) We can now complete the argument. The homotopy formula

$$
a_{k}=\int_{0}^{1} d t\left[C_{\alpha \beta}^{*} \frac{\delta^{L} a_{k}}{\delta C_{\alpha \beta}^{*}}+h_{\mu \nu \rho}^{*} \frac{\delta^{L} a_{k}}{\delta h_{\mu \nu \rho}^{*}}+h_{\mu \nu \rho} \frac{\delta^{L} a_{k}}{\delta h_{\mu \nu \rho}}\right]\left(t h, t h^{*}, t C^{*}\right)
$$

enables one to reconstruct $a_{k}$ from its E.L. derivatives. Inserting the expressions (4.39)-(4.41) for these E.L. derivatives, we get

$$
a_{k}=\delta\left(\int_{0}^{1} d t\left[C_{\alpha \beta}^{*} Z_{k-1}^{\prime \alpha \beta}+h_{\mu \nu \rho}^{*} X_{k}^{\prime \mu \nu \rho}+h_{\mu \nu \rho} Y_{k+1}^{\prime \mu \nu \rho}\right](t)\right)+\partial_{\rho} k^{\rho} .
$$

The first two terms in the argument of $\delta$ are manifestly invariant. To prove that the third term can be assumed to be invariant in Eq. (4.53) without loss of generality, we use Eq. (4.50) to find that

$$
h_{\mu \nu \rho} Y_{k+1}^{\prime \mu \nu \rho}=-\Psi_{k+1}^{\alpha \mu|\beta \nu| \gamma \rho} K_{\alpha \mu|\beta \nu| \gamma \rho}+G_{\alpha \beta \gamma} \widehat{X}_{k+1}^{\alpha \beta \gamma}+\partial_{\rho} \ell^{\rho}+\delta(\ldots),
$$

where we integrated by part thrice to get the first term of the r.h.s. while the hermiticity of $\mathcal{G}^{\mu \nu \rho \mid \alpha \beta \gamma}$ was used to obtain the second term.

We are left with $a_{k}=\delta \mu_{k+1}+\partial_{\rho} \nu_{k}^{\rho}$, where $\mu_{k+1}$ is invariant. That $\nu_{k}^{\rho}$ can now be chosen invariant is straightforward. Acting with $\gamma$ on the last equation yields $\partial_{\rho}\left(\gamma \nu_{k}^{\rho}\right)=0$. By the Poincaré lemma, $\gamma \nu_{k}^{\rho}=\partial_{\sigma}\left(\tau_{k}^{[\rho \sigma]}\right)$. Furthermore, Proposition 3 on $H(\gamma \mid d)$ for positive antighost number $k$ implies that one can redefine $\nu_{k}^{\rho}$ by the addition of trivial $d$-exact terms such that one can assume $\gamma \nu_{k}^{\rho}=0$. As the pureghost number of $\nu_{k}^{\rho}$ vanishes, the last equation implies that $\nu_{k}^{\rho}$ is an invariant polynomial. 


\section{Computation of deformations}

As explained in Section 3.1, nontrivial consistent interactions are in one-to-one correspondance with elements of $H^{n, 0}(s \mid d)$, i.e. solutions $a$ of the equation

$$
s a+d b=0,
$$

with form-degree $n$ and ghost number zero, modulo the equivalence relation

$$
a \sim a+s p+d q
$$

Quite generally, one can expand $a$ according to the antifield number, as

$$
a=a_{0}+a_{1}+a_{2}+\ldots a_{k}
$$

where $a_{i}$ has antifield number $i$. The expansion stops at some finite value of the antifield number by locality, as was proved in [36].

Let us recall [21] the meaning of the various components of $a$ in this expansion. The antifield-independent piece $a_{0}$ is the deformation of the Lagrangian; $a_{1}$, which is linear in the antifields $h^{* \mu \nu \rho}$, contains the information about the deformation of the gauge symmetries, given by the coefficients of $h^{* \mu \nu \rho} ; a_{2}$ contains the information about the deformation of the gauge algebra (the term $C^{*} C C$ gives the deformation of the structure functions appearing in the commutator of two gauge transformations, while the term $h^{*} h^{*} C C$ gives the on-shell closure terms); and the $a_{k}(k>2)$ give the informations about the deformation of the higher order structure functions and the reducibility conditions.

In fact, using the previous cohomological theorems and standard reasonings (see e.g. $[22]$ ), one can remove all components of $a$ with antifield number greater than 2 . The key point is that the invariant characteristic cohomology $H_{k}^{n, i n v}(\delta \mid d)$ controls the obstructions to the removal of the term $a_{k}$ from $a$ and that all $H_{k}^{n, i n v}(\delta \mid d)$ vanish for $k>2$ by Proposition 4 and Theorem 3 . This proves the first part of the following theorem:

Theorem 4. Let a be a local top form which is a nontrivial solution of the equation (5.54). Without loss of generality, one can assume that the decomposition (5.55) stops at antighost number two, i.e.

$$
a=a_{0}+a_{1}+a_{2} .
$$

If the last term $a_{2}$ is parity and Poincaré invariant, then it can always be written as the sum of

$$
a_{2}^{2}=f_{b c}^{a} C_{a}^{* \mu \nu}\left(T_{\mu \alpha \mid \beta}^{b} T_{\nu \alpha \mid \beta}^{c}-2 T_{\mu \alpha \mid \beta}^{b} T_{\nu \beta \mid \alpha}^{c}+\frac{3}{2} C^{b \alpha \beta} U_{\mu \alpha \mid \nu \beta}^{c}\right) d^{n} x
$$

and

$$
a_{2}^{4}=g_{b c}^{a} C_{a}^{* \mu \nu} U_{\mu \alpha \mid \beta \lambda}^{b} U_{\nu \alpha \mid \beta \lambda}^{c} d^{n} x
$$


where $f_{b c}^{a}$ and $g_{b c}^{a}$ are some arbitrary constant tensors that are antisymmetric under the exchange of $b$ and $c$. Notice that $a_{2}^{4}$ vanishes when $n=4$.

This most general parity and Poincaré invariant expression for $a_{2}$ is computed in Section 5.1 .

Let us note that the two components of $a_{2}$ do not contain the same number of derivatives: $a_{2}^{2}$ and $a_{2}^{4}$ contain respectively two and four derivatives. This implies that $a_{2}^{2}$ and $a_{2}^{4}$ lead to Lagrangian vertices with resp. three and five derivatives. The first kind of deformation (three derivatives) was studied in [6], however the case with five derivatives has never been considered before.

Similarly to (5.56), one can assume $b=b_{0}+b_{1}$. Inserting the expansions of $a$ and $b$ into (5.54) and decomposing $s$ as $s=\delta+\gamma$ yields

$$
\begin{array}{r}
\gamma a_{0}+\delta a_{1}+d b_{0}=0 \\
\gamma a_{1}+\delta a_{2}+d b_{1}=0 \\
\gamma a_{2}=0
\end{array}
$$

The general solution of (5.61) is given by Proposition 1. The computation of $a_{2}$ follows from the results obtained in Sections 4.4-4.6, applied to the equation (5.60).

Another consequence of the different number of derivatives in $a_{2}^{2}$ and $a_{2}^{4}$ is that the descents associated with both terms can be studied separately. Indeed, the operators appearing in the descent equations (5.59)-(5.61) are all homogeneous with respect to the number of derivatives, which means that one can split $a$ into eigenfunctions of the operator counting the number of derivatives and solve the equations separately for each of them. In the sequel we thus split the analysis: the descent starting from $a_{2}^{2}$ is analysed in Section 5.2, while the descent associated with $a_{2}^{4}$ is treated in Section 5.3.

\subsection{Most general term in antighost number two}

The equation (5.61) implies that, modulo trivial terms, $a_{2}=\alpha_{I} \omega^{I}$, where $\alpha_{I}$ is an invariant polynomial and the $\left\{\omega^{I}\right\}$ provide a basis of the polynomials in $C_{\mu \nu}, \widehat{T}_{\mu \nu \rho}, \widehat{U}_{\mu \nu \rho \sigma}$ (see Section 4.3). Let us stress that, as $a_{2}$ has ghost number zero and antifield number two, $\omega^{I}$ must have ghost number two.

Acting with $\gamma$ on (5.60) and using the triviality of $d$, one gets that $b_{1}$ should also be an element of $H(\gamma)$, i.e., modulo trivial terms, $b_{1}=\beta_{I} \omega^{I}$, where the $\beta_{I}$ are invariant polynomials.

Let us further expand $a_{2}$ and $b_{1}$ according to the $D$-degree defined in the proof of Propo- 
sition 3 in Section 4.4 :

$$
a_{2}=\sum_{i=0}^{M} a_{2}^{i}=\sum_{i=0}^{M} \alpha_{I_{i}} \omega^{I_{i}}, \quad b_{1}=\sum_{i=0}^{M} b_{1}^{i}=\sum_{i=0}^{M} \beta_{I_{i}} \omega^{I_{i}},
$$

where $a_{2}^{i}, b_{1}^{i}$ and $\omega^{I_{i}}$ have $D$-degree $i$. The equation (5.60) then reads

$$
\sum_{i} \delta\left[\alpha_{I_{i}} \omega^{I_{i}}\right]+\sum_{i} D\left[\beta_{I_{i}} \omega^{I_{i}}\right]=\gamma(\ldots)
$$

or equivalently

$$
\sum_{i} \delta\left[\alpha_{I_{i}}\right] \omega^{I_{i}}+\sum_{i} D_{0}\left[\beta_{I_{i}}\right] \omega^{I_{i}}+\sum_{i} \beta_{I_{i}} A_{I_{i+1}}^{I_{i}} \omega^{I_{i+1}}=\gamma(\ldots)
$$

where $A_{I_{i+1}}^{I_{i}} \omega^{I_{i+1}}=D \omega^{I_{i}}$, which implies

$$
\delta\left[\alpha_{I_{i}}\right]+D_{0}\left[\beta_{I_{i}}\right]+\beta_{I_{i-1}} A_{I_{i}}^{I_{i-1}}=0
$$

for each $D$-degree $i$, as the elements of the set $\left\{\omega^{I}\right\}$ are linearly independent nontrivial elements of $H(\gamma)$.

\section{D-degree decomposition:}

- degree zero : In $D$-degree 0 , the last equation reads $\delta\left[\alpha_{I_{0}}\right]+D_{0}\left[\beta_{I_{0}}\right]=0$, which implies that $\alpha_{I_{0}}$ belongs to $H_{2}(\delta \mid d)$. In antifield number 2, this group has nontrivial elements given by Proposition 5, which are proportional to $C_{a}^{* \mu \nu}$. The requirement of translation-invariance restricts the coefficient of $C_{a}^{* \mu \nu}$ to be constant. Indeed, it can be shown [39] that if the Lagrangian deformation $a_{0}$ is invariant under translations, then so are the other components of $a$. On the other hand, in $D$-degree 0 and ghost number 2 , we have $\omega^{I_{0}}=C_{\mu \rho}^{b} C_{\nu \sigma}^{c}$. To get a parity and Lorentz-invariant $a_{2}^{0}, \omega^{I_{0}}$ must be completed by multiplication with $C_{a}^{* \mu \nu}$ and some parity-invariant and covariantly constant tensor, i.e. a product of $\eta_{\mu \nu}$ 's. The only $a_{2}^{0}$ that can be thus built is $a_{2}^{0}=C_{a}^{* \mu \nu} C_{\mu \rho}^{b} C_{\nu}^{c \rho} f_{b c}^{a} d^{n} x$, where $f_{b c}^{a}$ is some constant tensor that parametrizes the deformation. From this expression, one computes that $b_{1}^{0}=\beta_{I_{0}} \omega^{I_{0}}=-3\left(h_{a}^{* \mu \nu \alpha}-\frac{1}{n} \eta^{\mu \nu} h_{a}^{* \alpha}\right) C_{\mu \rho}^{b} C_{\nu}^{c \rho} f_{b c}^{a} *\left(d x_{\alpha}\right)$, where $*\left(d x_{\alpha}\right)=\frac{1}{(n-1) !} \varepsilon_{\alpha \mu_{1} \ldots \mu_{n-1}} d x^{\mu_{1}} \ldots d x^{\mu_{n-1}}$.

- degree one : We now analyse Eq.(5.62) in D-degree 1, which reads

$$
\delta\left[\alpha_{I_{1}}\right]+D_{0}\left[\beta_{I_{1}}\right]+\beta_{I_{0}} A_{I_{1}}^{I_{0}}=0 .
$$

The last term can be read off $\beta_{I_{0}} A_{I_{1}}^{I_{0}} \omega^{I_{1}} \propto\left(h_{a}^{* \mu \nu \alpha}-\frac{1}{n} \eta^{\mu \nu} h_{a}^{* \alpha}\right) f_{b c}^{a} d^{n} x \widehat{T}_{\alpha(\mu \mid \rho)}^{b} C_{\nu}^{c \rho}$, and should be $\delta$-exact modulo $D_{0}$ for a solution of (5.63) to exist. However, the coefficient 
of $\widehat{T}_{\alpha(\mu \mid \rho)}^{b} C_{\nu}^{c \rho}$ is not $\delta$-exact modulo $D_{0}$. This is easily seen in the space of $x$-independent functions, as both $\delta$ and $D_{0}$ bring in one derivative while the coefficient contains none. As $\beta_{I_{0}}$ is allowed to depend explicitely on $x^{\mu}$, the argument is actually slightly more complicated: one must expand $\beta_{I_{0}}$ according to the number of derivatives of the fields in order to reach the conclusion. The detailed argument can be found in the proof of Theorem 7.3 in Ref. [38]. As $\beta_{I_{0}} A_{I_{1}}^{I_{0}}$ is not $\delta$-exact modulo $D_{0}$, it must vanish if (5.63) is to be satisfied. This implies that $f_{b c}^{a}$ vanishes, so that $a_{2}^{0}=0$ and $b_{1}^{0}=0$. One thus gets that $\alpha_{I_{1}}$ is an element of $H_{2}(\delta \mid d)$. However, there is no way to complete it in a Poincaré-invariant way because the only $\omega^{I_{1}}$ is $\omega^{I_{1}}=\widehat{T}_{\mu \nu \mid \rho}^{b} C_{\alpha \beta}^{c}$, which has an odd number of Lorentz indices, while $\alpha_{I_{1}} \propto C_{a}^{* \mu \nu}$ has an even number of them. Thus $a_{2}^{1}=0=b_{1}^{1}$.

- degree two : The equation (5.62) in $D$-degree 2 is then $\delta\left[\alpha_{I_{2}}\right]+D_{0}\left[\beta_{I_{2}}\right]=0$, which implies that $\alpha_{I_{2}}$ belongs to $H_{2}(\delta \mid d)$. One finds, most generally when $n>3$, that

$$
\begin{aligned}
& a_{2}^{2}=C_{a}^{* \mu \nu}\left(\widehat{T}_{\mu \alpha \mid \beta}^{b} \widehat{T}_{\nu}^{c \alpha \mid \beta} f_{[b c]}^{a}+\widehat{T}_{\mu \alpha \mid \beta}^{b} \widehat{T}_{\nu}^{c \beta \mid \alpha} g_{[b c]}^{a}+C^{b \alpha \beta} \widehat{U}_{\mu \alpha \mid \nu \beta}^{c} k_{b c}^{a}\right) d^{n} x, \\
& b_{1}^{2}=-3\left(h_{a}^{* \mu \nu \rho}-\frac{1}{n} \eta^{\mu \nu} h_{a}^{* \rho}\right)\left(\widehat{T}_{\mu \alpha \mid \beta}^{b} \widehat{T}_{\nu}^{c \alpha \mid \beta} f_{[b c]}^{a}+\widehat{T}_{\mu \alpha \mid \beta}^{b} \widehat{T}_{\nu}^{c \beta \mid \alpha} g_{[b c]}^{a}+C^{b \alpha \beta} \widehat{U}_{\mu \alpha \mid \nu \beta}^{c} k_{b c}^{a}\right) *\left(d x_{\rho}\right),
\end{aligned}
$$

where $f_{[b c]}^{a}, g_{[b c]}^{a}$ and $k_{b c}^{a}$ are three a priori independent constant tensors.

- degree three : Now, in the equation for $a_{2}^{3}$, we have

$$
\beta_{I_{2}} A_{I_{3}}^{I_{2}} \omega^{I_{3}} \propto\left[h_{a}^{* \mu \nu \rho} \widehat{U}_{\mu \alpha \mid \rho \beta}^{b} \widehat{T}_{\nu}^{c \alpha \mid \beta}\left(f_{[b c]}^{a}+g_{[b c]}^{a}-\frac{2}{3} k_{c b}^{a}\right)-\frac{1}{n} h_{a}^{* \rho} \widehat{U}_{\mu \alpha \mid \rho \beta}^{b} \widehat{T}^{c \mu \alpha \mid \beta}\left(f_{[b c]}^{a}+\frac{1}{2} g_{[b c]}^{a}\right)\right] d^{n} x,
$$

which implies, when $n>3$, that $g_{[b c]}^{a}=-2 f_{[b c]}^{a}$ and $k_{b c}^{a}=\frac{3}{2} f_{[b c]}^{a}$, since the coefficients of $\widehat{U}_{\mu \alpha \mid \rho \beta}^{b} \widehat{T}_{\nu}^{c \alpha \mid \beta}$ and $\widehat{U}_{\mu \alpha \mid \rho \beta}^{b} \widehat{T}_{\mu}^{c \alpha \mid \beta}$ are not $\delta$-exact modulo $D_{0}$. All this proves Equation (5.57), which is the expression $a_{2}^{2}$ found here modulo trivial terms. Provided that the above conditions are satisfied, $\alpha_{I_{3}}$ must be in $H_{2}(\delta \mid d)$. But no Poincaré-invariant $a_{2}^{3}$ can be built because $\omega^{I_{3}}=\widehat{T}_{\mu \alpha \mid \beta}^{b} \widehat{U}_{\nu \rho \mid \sigma \tau}^{c}$ has an odd number of Lorentz indices, so $a_{2}^{3}=0$.

- degree four : Repeating the same arguments for $a_{2}^{4}$, one gets $a_{2}^{4}=g^{a}{ }_{b c} C_{a}^{* \mu \nu}$ $\widehat{U}_{\mu \alpha \mid \beta \lambda}^{b} \widehat{U}_{\nu}^{c \alpha \mid \beta \lambda} d^{n} x$ and $b_{1}^{4}=-3\left(h_{a}^{* \mu \nu \rho}-\frac{1}{n} \eta^{\mu \nu} h_{a}^{* \rho}\right) \widehat{U}_{\mu \alpha \mid \beta \lambda}^{b} \widehat{U}_{\nu}^{c \alpha \mid \beta \lambda} g_{b c}^{a} *\left(d x_{\rho}\right)$, for some constant structure function $g_{b c}^{a}$. It is important to notice that $a_{2}^{4}$ vanishes in dimension $n=4$ because of the Schouten identity $0 \equiv C_{\mu_{1}}^{* \nu_{1}} \widehat{U}_{\mu_{2} \mu_{3} \mid}^{b}{ }_{\nu_{2} \nu_{3}} \widehat{U}_{\mu_{4} \mu_{5} \mid}^{c}{ }_{\nu_{4} \nu_{5}} \delta_{\left[\nu_{1}\right.}^{\left[\mu_{1}\right.} \ldots \delta_{\left.\nu_{5}\right]}^{\left.\mu_{5}\right]} \propto$ $C^{* \mu \nu} \widehat{U}_{\mu \alpha \mid \beta \lambda}^{b} \widehat{U}_{\nu}^{c \alpha \mid \beta \lambda}$. No condition is imposed on $g_{b c}^{a}$ by equations in higher $D$-degree because $D_{1} b_{1}^{4}=0$. This proves Equation (5.58).

- degree $>4$ : Finally, there are no $a_{2}^{i}$ for $i>4$ because there is no ghost combination $\omega^{I_{i}}$ of ghost number two and $D$-degree higher than four.

Summarizing, we have proved the second part of Theorem 4. 


\subsection{Berends-Burgers-van Dam's deformation}

In this section, we consider the deformation related to $a_{2}^{2}$ given by (5.57). As explained above, $a_{2}=a_{2}^{2}$ must now be completed into a solution $a$ of $s a+d b=0$ by adding terms with lower antifield number. The complete solution $a$ provides then the first-order deformation term $W_{1}=\int a$ of an interacting theory. The next step is to check that higher order terms $W_{2}, W_{3}$, etc. can be built to get the full interacting theory.

In the case considered here, we show that a first-order interaction term $W_{1}$ can be constructed; however, there is an obstruction to the existence of $W_{2}$, which prevents its completion into a consistent interacting theory.

\subsubsection{Existence of a first-order deformation}

In this section, the descent equations (5.59) and (5.60), i.e. $\gamma a_{0}+\delta a_{1}+d b_{0}=0$ and $\gamma a_{1}+\delta a_{2}+d b_{1}=0$, are solved for $a_{1}$ and $a_{0}$.

The latter of these equations admits the particular solution

$$
\begin{array}{r}
a_{1}^{p}=-\frac{3}{2}\left[\left(h_{a}^{* \mu \nu \rho}-\frac{1}{n} \eta^{\mu \nu} h_{a}^{* \rho}\right)\left(2 \partial_{[\mu} h_{\alpha] \beta \rho}^{b}\left(T_{\nu \alpha \mid \beta}^{c}-2 T_{\nu \beta \mid \alpha}^{c}\right)+h_{\alpha \beta \rho}^{b} U_{\mu \alpha \mid \nu \beta}^{c}-3 C^{b \alpha \beta} \partial_{[\nu} h_{\beta] \rho[\alpha, \mu]}^{c}\right)\right. \\
\left.+\frac{1}{n} h_{a}^{* \rho} T_{\rho \alpha \mid \beta}^{b}\left(\partial_{\sigma} h^{c \sigma \alpha \beta}-\partial^{\alpha} h^{c \beta}-\partial^{\beta} h^{c \alpha}\right)\right] f_{b c}^{a} d^{n} x .
\end{array}
$$

To this particular solution, one must add the general solution $\bar{a}_{1}$ of $\gamma \bar{a}_{1}+d b_{1}=0$, or equivalently (by Proposition 3) of $\gamma \bar{a}_{1}=0$. In ghost number zero, antifield number one and with two derivatives, this solution is, modulo trivial $\delta$-, $\gamma$-and $d$-exact terms,

$$
\bar{a}_{1}=h_{\mu \nu \rho}^{* a} G_{\sigma}^{b \mu \nu} C^{c \rho \sigma} l_{(a b) c}^{1}+h_{\mu}^{* a} G_{\nu}^{b} C^{c \mu \nu} l_{(a b) c}^{2}+h^{* a \mu} G_{\mu \nu \rho}^{b} C^{c \nu \rho} l_{a b c}^{3},
$$

where $l_{(a b) c}^{1}, l_{(a b) c}^{2}$ and $l_{a b c}^{3}$ are some arbitrary constants. For future convenience, we also add to $a_{1}^{p}+\bar{a}_{1}$ the trivial term $\gamma b_{1}$ where

$$
\begin{aligned}
b_{1}= & f_{b c}^{a} h_{a \mu \nu \rho}^{*}\left(-\frac{3}{2} h^{b \mu \sigma \tau} \partial^{\nu} h_{\sigma \tau}^{c \rho}-2 h^{b \mu \sigma \tau} \partial_{\sigma} h_{\tau}^{c \nu \rho}+3 h^{b \mu} \partial^{\nu} h^{c \rho}-3 h_{\sigma}^{b} \partial^{\mu} h^{c \nu \rho \sigma}+2 h_{\sigma}^{b} \partial^{\sigma} h^{c \mu \nu \rho}\right) \\
& +f_{a b c} h_{\mu}^{* a}\left(2 h^{b \mu \nu \rho} \partial_{\nu} h_{\rho}^{c}-h^{b \mu \nu \rho} \partial^{\sigma} h_{\nu \rho \sigma}^{c}+3 h^{b \mu} \partial^{\sigma} h_{\sigma}^{c}-\frac{1}{2} h_{\nu \rho \sigma}^{b} \partial^{\mu} h^{c \nu \rho \sigma}+6 h_{\nu}^{b} \partial_{\rho} h^{c \mu \nu \rho}\right) .
\end{aligned}
$$

In short, up to trivial terms, the most general $a_{1}$, solution of $\gamma a_{1}+\delta a_{2}+d b_{1}=0$, is $a_{1}=a_{1}^{p}+\bar{a}_{1}+\gamma b_{1}$.

The next step is to find $a_{0}$ such that $\gamma a_{0}+\delta a_{1}+d b_{0}=0$. A cumbersome but straightforward computation shows that necessary (and, as we will see, sufficient) conditions for a solution $a_{0}$ to exist are (i) $f_{[b c]}^{a}$ is totally antisymmetric, or more precisely $\delta_{a d} f_{[b c]}^{d}=f_{[a b c]}$, (ii) $l_{(a b) c}^{1}=l_{(a b) c}^{2}=0$ and (iii) $l_{a b c}^{3}=-\frac{9}{8} f_{[a b c]}$. This computation follows the lines of an argument developped in [22], which considers the most general $a_{0}$ and matches the coefficients of the 
terms with the structure $C h^{\prime} h^{\prime}$, where $h^{\prime}$ denotes the trace of $h$. In four dimensions, one must take into account that some of these terms are related by Schouten identities; however, this does not change the conclusions. Once the conditions (i) to (iii) are satisfied, one can explicitly build the solution $a_{0}$, which corresponds to the spin-3 vertex found in [6] in which the structure function $f_{a b c}$ has been replaced by $-\frac{3}{8} f_{a b c}$. The deformation $a_{0}$ of the Lagrangian can be found in the appendix A. It is unique up to solutions $\bar{a}_{0}$ of the homogeneous equation $\gamma \bar{a}_{0}+d b_{0}=0$.

We have thus proved by a new method that the spin-3 vertex of [6] is the only consistent nontrivial first-order deformation of the free spin-3 theory with at most ${ }^{10}$ three derivatives in the Lagrangian, modulo deformations $\bar{a}_{0}$ of the latter that are gauge-invariant up to a total derivative, i.e. such that $\gamma \bar{a}_{0}+d b_{0}=0$. However, as is known from [9], this deformation cannot be completed to all orders, as is proved again in the next section.

\subsubsection{Obstruction for the second-order deformation}

In the previous section, we have constructed a first-order deformation $W_{1}=\int\left(a_{0}+a_{1}+a_{2}\right)$ of the free functional $W_{0}$. As explained in Section 4.2, a consistent second-order deformation $W_{2}$ must satisfy the condition

$$
\left(W_{1}, W_{1}\right)_{a . b .}=-2 s W_{2}
$$

Expanding $\left(W_{1}, W_{1}\right)_{a . b .}$ according to the antifield number, one finds

$$
\left(W_{1}, W_{1}\right)_{a . b .}=\int d^{n} x\left(\alpha_{0}+\alpha_{1}+\alpha_{2}\right)
$$

where the term of antifield number two $\alpha_{2}$ comes from the antibracket of $a_{2}$ with itself.

If one also expands $W_{2}$ according to the antifield number, one gets from (5.64) the following condition on $\alpha_{2}$ (it is easy to see that the expansion of $W_{2}$ can be assumed to stop at antifield number three, $W_{2}=\int d^{n} x\left(c_{0}+c_{1}+c_{2}+c_{3}\right)$ and that $c_{3}$ may be assumed to be invariant, $\left.\gamma c_{3}=0\right)$

$$
\alpha_{2}=-2\left(\gamma c_{2}+\delta c_{3}\right)+\partial_{\mu} b_{2}^{\mu}
$$

Explicitly,

$$
\begin{aligned}
\alpha_{2}=\frac{1}{2} f_{a b c} f_{d e}^{c} C_{\mu \nu}^{* a} & \left(-4 \widehat{T}^{b \mu \alpha \mid \beta} \widehat{T}^{d \nu \rho \mid \sigma} \widehat{U}_{\alpha \rho \mid \beta \sigma}^{e}+5 \widehat{T}^{b \mu \alpha \mid \beta} \widehat{T}^{d \nu \rho \mid \sigma} \widehat{U}_{\alpha \sigma \mid \beta \rho}^{e}-3 \widehat{T}^{b \mu \alpha \mid \beta} \widehat{T}_{\alpha \rho \mid \sigma}^{d} \widehat{U}_{\beta}^{e \sigma \nu \mid \rho}\right. \\
& +\widehat{T}^{b \mu \alpha \mid \beta} \widehat{T}_{\beta \rho \mid \sigma}^{d} \widehat{U}_{\alpha}^{e \rho \nu \mid \sigma}+\widehat{T}^{b \mu \alpha \mid \beta} \widehat{T}_{\beta \rho \mid \sigma}^{d} \widehat{U}_{\alpha}^{e \sigma \nu \mid \rho}-\frac{3}{2} \widehat{U}^{b \mu \alpha \mid \nu \beta} \widehat{T}_{\alpha \rho \mid \sigma}^{d} \widehat{T}_{\beta}^{e \rho \mid \sigma}
\end{aligned}
$$

\footnotetext{
${ }^{10}$ The developments above prove the three-derivatives case. For less derivatives, it follows from above that $a_{2}=0$, which implies that $\gamma a_{1}=0$ by $(5.60)$; however there is no such parity and Poincaré-invariant nontrivial $a_{1}$ with less than two derivatives, so $a_{1}=0$ as well.
} 


$$
\begin{aligned}
& +3 \widehat{U}^{b \mu \alpha \mid \nu \beta} \widehat{T}_{\alpha \rho \mid \sigma}^{d} \widehat{T}_{\beta}^{e \sigma \mid \rho}+\frac{9}{4} \widehat{U}^{b \mu \alpha \mid \nu \beta} C^{d \rho \sigma} \widehat{U}_{\alpha \sigma \mid \beta \rho}^{e}+\frac{3}{2} C_{\alpha \beta}^{b} \widehat{U}^{d \rho \mu \mid \sigma \alpha} \widehat{U}_{\rho}^{e \nu \mid \beta}{ }_{\sigma} \\
& \left.-\frac{3}{4} C_{\alpha \beta}^{b} \widehat{U}^{d \rho \mu \mid \sigma \alpha} \widehat{U}_{\sigma}^{e \nu \mid \beta}{ }_{\rho}+\frac{3}{4} C^{b \alpha \beta} \widehat{U}_{\rho \alpha \mid \sigma \beta}^{d} \widehat{U}^{e \rho \mu \mid \sigma \nu}\right)+\gamma(\ldots) .
\end{aligned}
$$

It is impossible to get an expression with three ghosts, one $C^{*}$ and no fields, by acting with $\delta$ on $c_{3}$, so we can assume without loss of generality that $c_{3}$ vanishes, which implies that $\alpha_{2}$ should be $\gamma$-exact modulo total derivatives.

However, $\alpha_{2}$ is not a mod- $d \gamma$-coboundary unless it vanishes. Indeed, suppose we have

$$
\alpha_{2}=\gamma(u)+\partial_{\mu} k^{\mu}
$$

Both $u$ and $k^{\mu}$ have antifield number two and we can restrict ourselves to their components linear in $C^{*}$ without loss of generality (so that the gauge algebra closes off-shell at second order). We can also assume that $u$ contains $C^{*}$ undifferentiated, since derivatives can be removed through integration by parts. As the Euler derivative of a divergence is zero, we can reformulate the question as to whether the following identity holds,

$$
\frac{\delta^{L} \alpha_{2}}{\delta C_{\mu \nu}^{* a}}=\frac{\delta^{L}(\gamma u)}{\delta C_{\mu \nu}^{* a}}=-\gamma\left(\frac{\partial^{L} u}{\partial C_{\mu \nu}^{* a}}\right) .
$$

since $\gamma C^{*}=0$ and $C^{*}$ appears undifferentiated in $u$. On the other hand, $\frac{\delta^{L} \alpha_{2}}{\delta C_{\mu \nu}^{* a}}$ is a sum of nontrivial elements of $H(\gamma)$; it can be $\gamma$-exact only if it vanishes. Consequently, a necessary condition for the closure of the gauge transformations $\left(c_{2}\right.$ may be assumed to be linear in the antifields) is $\alpha_{2}=0$.

Finally, $\alpha_{2}$ vanishes if and only if $f_{a b c} f_{d e}^{c}=0$ (nilpotency of the algebra) or $n=3$, which implies when $n>3$ the vanishing of $f_{a b c}$ (by Lemma 1), and thus of the whole deformation candidate.

Let us note that originally, in the work [9], the obstruction to this first-order deformation appeared under the weaker form $f_{a b c} f_{d e}^{c}=f_{a d c} f_{b e}^{c}$ (associativity) and was obtained by demanding the closure of the algebra of gauge transformations at second order in the deformation parameter.

\subsection{Five-derivative deformation}

We now consider the deformation related to $a_{2}=a_{2}^{4}$, written in Equation (5.58). In this case, the general solution $a_{1}$ of $\gamma a_{1}+\delta a_{2}+d b_{1}=0$ is, modulo trivial terms,

$$
a_{1}=-2\left(h_{a}^{* \mu \nu \rho}-\frac{1}{n} \eta^{\mu \nu} h_{a}^{* \rho}\right) \partial_{[\mu} h_{\alpha] \rho[\beta, \lambda]}^{b} U_{\nu}^{c \alpha \mid \beta \lambda} g_{[b c]}^{a} d^{n} x+\bar{a}_{1},
$$

where $\bar{a}_{1}$ is an arbitrary element of $H(\gamma)$. 
When the structure constant is completely antisymmetric in its indices, a Lagrangian deformation $a_{0}$ such that $\gamma a_{0}+\delta a_{1}+d b_{0}=0$ can be computed. However, its expression is quite long and is therefore to be found in the appendix B. We used the symbolic manipulation program FORM [40] for its computation. This nontrivial first-order deformation of the free theory had not been found in the previous spin-three analyzes in Minkowski space-time, which is related to the assumption usually made that the Lagrangian deformation should contain at most three derivatives, while it contains five of them in this case.

However, it would be very interesting to see whether the cubic vertex written in Appendix $\mathrm{B}$ could be related to the flat space limit of the higher-spin vertices of the second reference of [18]. At first order in the deformation parameter, it might be possible to take some flat space limit of the $(A) d S_{n}$ cubic vertices. A very appropriate free limit must indeed be taken: The dimensionless coupling constant $g$ of full higher-spin gauge theory should go to zero in a way that compensates the non-analyticity $\sim 1 / \Lambda^{m}$ in the cosmological constant $\Lambda$ of the cubic vertices, i.e. such that the ratio $g / \Lambda^{m}$ is finite. The vertex could then be recovered in such appropriate limits from the action of [41] by substituting the linearized spin-3 field strengths for the full nonlinear ones at quadratic order and replacing the auxiliary and extra connections by their expressions in terms of the spin-3 gauge field obtained by solving the linearized torsion-like constaints, as explained in [2,3,18] (and references therein). Such a relation would provide a geometric meaning for the complicated expression of Appendix B.

The next step is to find the second order components of the deformation. Similarly to the previous case, it can easily be checked that we can assume $c_{3}=0$. However, no obstruction arises from the constraint $\alpha_{2} \equiv\left(a_{2}, a_{2}\right)=-2 \gamma c_{2}+\partial_{m} k^{\mu}$. If this candidate for an interacting theory is obstructed, the obstructions should arise at some later stage, i.e. beyond the (possibly on-shell) closure of the gauge transformations.

For completeness, one should check if $\gamma a_{0}+\delta a_{1}+d b_{0}=0$ admits a solution $a_{0}$ when the structure constant $g_{b c}^{d}=g_{[b c]}^{d}$ is not completely antisymmetric but has the "hook" symmetry property $\delta_{d[a} g_{b c]}^{d}=0$. However, the computations involved are very cumbersome and we were not able to reach any conclusion about the existence of such an $a_{0}$.

\section{Conclusions and perspectives}

In this paper we carefully analyzed the problem of introducing consistent interactions among a countable collection of spin-3 gauge fields in flat space-time of arbitrary dimension $n>$ 3. For this purpose we used the powerful BRST cohomological deformation techniques in order to be as exhaustive as possible. Under the sole assumptions of locality, parity invariance, Poincaré invariance and perturbative deformation of the free theory, we proved 
that only two classes of non-abelian gauge symmetries are consistent at first order. They close off-shell and are entirely characterized by the structure constants of some internal anticommutative algebra (as for Yang-Mills's theories). When these constant tensors are completely antisymmetric (this is possible only for a set of different massless spin-3 fields), there exist actions that are invariant at first order under the non-Abelian gauge symmetries. The first deformation corresponds to the well-known Berends-Burgers-van Dam cubic vertex which involves three derivatives of the fields and becomes inconsistent at second order. The second deformation is defined for $n>4$ and corresponds to a cubic vertex that involves five derivatives. There are some indications that this deformation could be obtained from an appropriate flat-space limit of the nonlinear $(A) d S_{n}$ higher-spin gauge theory of Ref. [2].

The antisymmetry condition $g_{a b c}=g_{[a b c]}$ on the structure constant of the second deformation is only sufficient for the existence of the vertex. It would be interesting to establish whether a constant tensor $g_{[b c]}^{a}$ with the "hook" symmetries $\delta_{d[a} g_{b c]}^{d}=0$ might not also give rise to a consistent first-order vertex. If this first-order non-abelian deformation turned

out to exist, then there would be no other one, under the assumptions stated above. The relaxation of the parity symmetry requirement and the special case $n=3$ also deserve more study [42].

Moreover, it would be of prime importance to investigate whether the second first-order consistent deformation could be extended to higher orders in the deformation parameter. At second order, a first test has been passed where the Berends-Burgers-van Dam vertex fails, but unfortunately the lengthy nature of the five-derivative cubic vertex makes further analysis very tedious.

Last but not least, it would be of interest to enlarge the set of fields to spin 2, 3 and 4 and see if this allows to remove the previous obstruction at order two. A hint that this might be sufficient comes from the fact that the commutator of two spin-3 generators produces spin-2 and spin-4 generators for the bosonic higher-spin algebra of Ref. [2].

\section{Acknowledgments}

N. B. thanks S. Leclercq for discussions. X. B. acknowledges T. Damour for interesting comments.

The work of S.C. is supported in part by the "Interuniversity Attraction Poles Programme - Belgian Science Policy" and by IISN-Belgium (convention 4.4505.86). Moreover, X.B. and S.C. are supported in part by the European Commission FP6 programme MRTN-CT-2004005104, in which S.C. is associated to the V.U.Brussel (Belgium). 


\section{Appendices}

In this appendix, we provide the Lagrangian deformations $a_{0}$ for the first-order interactions found in Section 5, as well as the first-order deformation of the gauge transformations for the Berends-Burgers-van Dam vertex.

\section{A Three-derivative vertex}

The deformation

$$
\int a_{0}=f_{[a b c]} S^{a b c} ; \quad S^{a b c}\left[h_{\mu \nu \rho}^{d}\right]=-\frac{3}{8} \int \mathcal{L}_{B B v D}^{a b c} d^{n} x
$$

related to the element $a_{2}^{2}$ of Section 5.2 is the Berends-Burgers-van Dam cubic vertex

$$
\begin{aligned}
& \mathcal{L}_{B B v D}^{a b c}=-\frac{3}{2} h^{a \alpha} h^{b \beta, \gamma} h_{\beta, \alpha \gamma}^{c}+3 h^{a \alpha, \beta} h^{b \gamma} h_{\gamma, \alpha \beta}^{c}+6 h^{a \alpha \beta \gamma, \delta} h_{\alpha}^{b} h_{\beta, \gamma \delta}^{c}+\frac{1}{2} h^{a \alpha} h^{b \beta \gamma \delta, \varepsilon} h_{\beta \gamma \delta, \alpha \varepsilon}^{c} \\
& +h^{a \alpha}{ }_{, \alpha \beta} h_{\gamma \delta \varepsilon}^{b} h^{c \gamma \delta \varepsilon, \beta}+h^{a \alpha, \beta} h^{b \gamma \delta \varepsilon} h_{\gamma \delta \varepsilon, \alpha \beta}^{c}-3 h_{\alpha \beta \gamma}^{a} h_{\delta, \varepsilon}^{b \alpha \beta} h^{c \delta, \gamma \varepsilon}-3 h_{\alpha \beta \gamma}^{a} h^{b \alpha \beta \delta, \gamma \varepsilon} h_{\delta, \varepsilon}^{c} \\
& +3 h_{\alpha \beta \gamma, \delta}^{a} h^{b \alpha \beta \varepsilon} h_{\varepsilon}^{c, \gamma \delta}+3 h_{\alpha \beta \gamma}^{a}{ }^{, \gamma \delta} h^{b \alpha \beta \varepsilon} h_{\varepsilon, \delta}^{c}-\frac{9}{4} h_{\alpha, \beta \gamma}^{a} h^{b \beta} h^{c \gamma, \alpha}-\frac{1}{4} h_{\alpha, \beta}^{a} h^{b \beta, \gamma} h_{\gamma}^{c, \alpha} \\
& -3 h_{\alpha \beta \gamma}^{a} h^{b \delta, \alpha} h_{\delta}^{c, \beta \gamma}-\frac{3}{2} h_{\alpha}^{a, \alpha} h^{b \beta, \gamma} h_{\beta \gamma \delta}^{c}{ }^{, \delta}+3 h_{\alpha}^{a} h_{\beta, \gamma}^{b} h_{\delta}^{c \beta \gamma, \alpha \delta}+\frac{3}{2} h_{\alpha}^{a, \alpha \beta} h^{b \gamma, \delta} h_{\beta \gamma \delta}^{c} \\
& +3 h_{\alpha, \beta}^{a} h_{\gamma, \delta}^{b} h^{c \beta \gamma \delta, \alpha}-\frac{3}{2} h_{\alpha}^{a} h_{\beta \gamma \delta}^{b}{ }^{, \beta} h_{\varepsilon}^{c \gamma \delta, \alpha \varepsilon}-6 h_{\alpha \beta \gamma}^{a}{ }^{, \alpha \delta} h^{b \beta, \varepsilon} h_{\delta \varepsilon}^{c} \gamma \\
& +6 h_{\alpha \beta \gamma}^{a}{ }^{, \alpha \delta} h^{b \beta} h_{\delta \varepsilon}^{c}{ }^{\gamma, \varepsilon}-2 h_{\alpha \beta \gamma, \delta}^{a} h_{\lambda}^{b \alpha \delta, \varepsilon} h_{\varepsilon}^{c \lambda \beta, \gamma}+h_{\alpha \beta \gamma}^{a} h_{\delta \varepsilon \lambda}^{b}{ }^{, \alpha} h^{c \delta \varepsilon \lambda, \beta \gamma} \\
& -3 h_{\alpha \beta \gamma}^{a}{ }^{\alpha} h_{\delta}^{b \beta \gamma, \varepsilon} h_{\varepsilon \lambda}^{c} \delta, \lambda+3 h_{\alpha \beta \gamma}^{a}{ }^{, \alpha \delta} h^{b \beta \gamma \varepsilon, \lambda} h_{\varepsilon \delta \lambda}^{c}+6 h_{\alpha \beta \gamma, \delta}^{a} h^{b \alpha \beta \varepsilon, \lambda} h_{\varepsilon \lambda}^{c \delta, \gamma},
\end{aligned}
$$

where we remind that indices after a coma denote partial derivatives.

The first-order deformation of the gauge transformations is given by

$$
\delta_{\lambda}^{1} h_{\mu \nu \rho}^{a}=f_{b c}^{a} \Phi_{\mu \nu \rho}^{b c}
$$

where $\Phi_{\mu \nu \rho}^{b c}$ is the completely symmetric component of

$$
\begin{aligned}
\phi_{\mu \nu \rho}^{b c}=\quad & 6 h^{b \sigma} \lambda_{\mu \sigma, \nu \rho}^{c}-3 h^{b \sigma} \lambda_{\mu \nu, \rho \sigma}^{c}+6 h_{\mu, \nu}^{b} \lambda_{\sigma \rho}^{c, \sigma}-6 h_{\mu}^{b} \lambda_{\sigma \nu, \rho}^{c}{ }^{\sigma}-\frac{15}{4} h_{\mu \sigma \tau, \nu}^{b} \lambda_{\rho}^{c \sigma, \tau} \\
& +\frac{31}{4} h_{\mu}^{b \sigma \tau} \lambda_{\nu \sigma, \tau \rho}^{c}+\frac{9}{4} h_{\mu \nu \sigma, \rho \tau}^{b} \lambda^{c \sigma \tau}-\frac{11}{2} h_{\mu \nu}^{b}{ }^{\sigma, \tau} \lambda_{\sigma(\tau, \rho)}^{c} \\
& -6 h_{\mu \nu \sigma, \rho}^{b} \lambda_{, \tau}^{c \sigma \tau}-\frac{3}{4} h_{\mu \sigma \tau, \nu}^{b} \lambda_{, \rho}^{c \sigma \tau}-\frac{9}{8} h_{\mu \sigma \tau, \nu \rho}^{b} \lambda^{c \sigma \tau}+\frac{9}{8} h_{\mu}^{b \sigma \tau} \lambda_{\sigma \tau, \nu \rho}^{c} \\
& -\frac{1}{2} h_{\mu \nu \sigma, \tau}^{b} \lambda_{\rho}^{c \tau, \sigma}+\frac{13}{8} h_{\mu}^{b \sigma \tau} \lambda_{\nu \rho, \sigma \tau}^{c}+4 h_{\mu \nu \rho, \sigma}^{b} \lambda_{, \tau}^{c \sigma \tau}-\frac{9}{8} h_{\mu \nu \rho}^{b}{ }_{, \sigma \tau} \lambda_{\sigma \tau}^{c} \\
+ & \eta_{\mu \nu}\left(\begin{array}{l}
\frac{9}{4}\left(h^{b \sigma, \tau} \lambda_{\rho \sigma}^{c}-h_{\sigma, \rho \tau}^{b} \lambda^{c \sigma \tau}-h_{\eta \sigma \tau}^{b}{ }_{, \eta \sigma} \lambda_{\rho}^{c \tau}\right)+\frac{9}{8}\left(h_{\sigma}^{b, \sigma \tau} \lambda_{\rho \tau}^{c}+h_{, \eta \rho}^{b \eta \sigma \tau}{ }_{\sigma \tau}^{c}\right)
\end{array}\right.
\end{aligned}
$$




$$
\begin{aligned}
& +6\left(h_{\sigma}^{b, \sigma} \lambda_{\rho \tau}^{c, \tau}-h^{b \sigma} \lambda_{\sigma \tau, \rho}^{c}{ }^{\tau}-h^{b \sigma} \lambda_{\sigma \rho, \tau}^{c}{ }^{\tau}-h_{\sigma}^{b} \lambda_{\rho \tau}^{c}{ }^{, \sigma \tau}-h_{\rho}^{b} \lambda_{\sigma \tau}^{c, \sigma \tau}+2 h_{\rho \sigma \tau}^{b}{ }^{, \sigma} \lambda_{\eta}^{c \tau, \eta}\right) \\
& +\frac{3}{2}\left(h^{b \eta \sigma \tau} \lambda_{\sigma \tau, \eta \rho}^{c}-h_{\eta \sigma \tau, \rho}^{b} \lambda^{c \sigma \tau, \eta}\right)+\left(1-\frac{3}{4 n}\right)\left(2 h^{b \sigma, \tau} \lambda_{\sigma \tau, \rho}^{c}-h_{\eta}^{b \sigma \tau, \eta} \lambda_{\sigma \tau, \rho}^{c}\right) \\
& +\left(2+\frac{3}{4 n}\right)\left(h_{\sigma, \tau}^{b} \lambda_{\rho}^{c \sigma, \tau}+h_{\sigma, \tau}^{b} \lambda_{\rho}^{c \tau, \sigma}-h_{\sigma}^{b \tau \eta, \sigma} \lambda_{\rho \tau, \eta}^{c}-h_{\rho \sigma \tau}^{b} \lambda_{\eta}^{c \sigma, \tau \eta}+\frac{1}{2} h_{\rho}^{b \sigma \tau} \lambda_{\sigma \tau, \eta}^{c} \eta\right) \\
& \left.+\frac{9}{8}\left(1-\frac{1}{n}\right)\left(-h_{\rho \sigma \tau, \eta}^{b} \lambda^{c \sigma \tau}+2 h_{\rho \sigma}^{b \tau, \eta \sigma} \lambda_{\eta \tau}^{c}-h_{\rho}^{b, \sigma \tau} \lambda_{\sigma \tau}^{c}\right)\right) .
\end{aligned}
$$

This expression is equivalent to that of [6] modulo field redefinitions.

\section{B Five-derivative vertex}

In this appendix, we give the deformation $a_{0}$ related to the element $a_{2}^{4}$ of Section 5.3 with completely antisymmetric structure constants. It satisfies the equation $\gamma a_{0}+\delta a_{1}+d b_{0}=0$ for $a_{1}$ defined by (5.66), in which $\bar{a}_{1}=0$. The deformation is

$$
\int a_{0}=g^{[a b c]} T_{a b c} ; \quad T_{a b c}\left[h_{\mu \nu \rho}^{d}\right]=\frac{1}{2} \int \mathcal{L}_{a b c} d^{n} x
$$

where

$$
\begin{aligned}
& \mathcal{L}_{a b c}= \\
& h_{a}^{\mu \nu \rho}\left(-\frac{7}{4} \partial_{\mu \nu} h_{b}^{\lambda \sigma \tau} \partial_{\rho \sigma \tau} h_{c \lambda}-\frac{1}{4} \partial_{\mu \nu} h_{b}^{\lambda \sigma \tau} \partial_{\rho \eta} \partial^{\eta} h_{c \lambda \sigma \tau}-\frac{1}{2} \partial_{\mu \nu} h_{b}^{\lambda} \partial_{\rho \lambda \sigma} h_{c}^{\sigma}-\frac{3}{4} \partial_{\mu \nu} h_{b}^{\lambda} \partial_{\rho \sigma \tau} h_{c \lambda}^{\sigma \tau}\right. \\
& -\frac{5}{3} \partial_{\mu} h_{b}^{\lambda \sigma \tau} \partial_{\nu \rho \lambda \eta} h_{c \sigma \tau}^{\eta}+\frac{1}{2} \partial_{\mu} h_{b}^{\lambda \sigma \tau} \partial_{\nu \rho \eta} \partial^{\eta} h_{c \lambda \sigma \tau}+\frac{2}{3} \partial_{\mu} h_{b}^{\lambda} \partial_{\nu \rho \sigma \tau} h_{c \lambda}{ }^{\sigma \tau}-\frac{4}{3} \partial_{\mu} h_{b}^{\lambda} \partial_{\nu \rho \sigma} \partial^{\sigma} h_{c \lambda} \\
& +\frac{5}{4} \partial_{\sigma \tau} h_{b}^{\sigma \tau \lambda} \partial_{\mu \nu \rho} h_{c \lambda}-\frac{5}{3} \partial_{\sigma \tau} h_{b}^{\sigma \lambda \eta} \partial_{\mu \nu \rho} h_{c \lambda \eta}^{\tau}+\frac{3}{4} \partial_{\sigma} \partial^{\sigma} h_{b}^{\lambda \eta \tau} \partial_{\mu \nu \rho} h_{c \lambda \eta \tau}+\frac{1}{2} \partial_{\sigma \tau} h_{b}^{\sigma} \partial_{\mu \nu \rho} h_{c}^{\tau} \\
& +\frac{23}{12} \partial_{\sigma \tau} h_{b}^{\lambda} \partial_{\mu \nu \rho} h_{c \lambda}{ }^{\sigma \tau}-\frac{4}{3} \partial_{\sigma} \partial^{\sigma} h_{b}^{\lambda} \partial_{\mu \nu \rho} h_{c \lambda}-\frac{51}{16} \partial_{\mu \nu} h_{b \rho} \partial_{\sigma \tau} \partial^{\sigma} h_{c}^{\tau}-\frac{11}{8} \partial_{\mu} h_{b \nu}{ }^{\sigma \tau} \partial_{\rho \sigma \tau \lambda} h_{c}^{\lambda} \\
& +\frac{5}{4} \partial_{\mu} h_{b \nu \sigma \tau} \partial_{\rho \lambda \eta} \partial^{\tau} h_{c}^{\sigma \lambda \eta}-\frac{3}{8} \partial_{\mu} h_{b \nu \sigma \tau} \partial_{\rho \lambda} \partial^{\lambda \tau} h_{c}^{\sigma}+\frac{9}{4} \partial_{\mu} h_{b \nu \sigma \tau} \partial_{\rho \lambda \eta} \partial^{\eta} h_{c}^{\sigma \tau \lambda}-\frac{1}{12} \partial_{\mu} h_{b \nu} \partial_{\rho \lambda \sigma \tau} h_{c}^{\lambda \sigma \tau} \\
& -\frac{3}{2} \partial_{\mu} h_{b \nu} \partial_{\rho \lambda \sigma} \partial^{\sigma} h_{c}^{\lambda}-\frac{11}{16} \partial_{\lambda} h_{b \mu}^{\sigma \tau} \partial_{\nu \rho \sigma \tau} h_{c}^{\lambda}-\frac{1}{4} \partial_{\lambda \eta} h_{b \mu \sigma \tau} \partial_{\nu \rho} \partial^{\tau} h_{c}^{\lambda \eta \sigma}+\frac{3}{4} \partial_{\lambda} \partial^{\lambda} h_{b \mu \sigma}^{\tau} \partial_{\nu \rho \tau} h_{c}^{\sigma} \\
& +\frac{7}{4} \partial_{\eta \lambda} h_{b \mu} \partial_{\nu \rho} \partial^{\eta} h_{c}{ }^{\lambda}-\frac{19}{16} \partial_{\eta} \partial^{\eta} h_{b \mu} \partial_{\nu \rho \lambda} h_{c}{ }^{\lambda}+\frac{11}{4} \partial_{\mu \lambda} h_{b \nu}{ }^{\lambda \sigma} \partial_{\sigma \tau \eta} h_{c \rho}{ }^{\tau \eta}+\frac{3}{4} \partial_{\mu} h_{b \nu \sigma \tau} \partial^{\sigma \tau \lambda \eta} h_{c \rho \lambda \eta} \\
& +\frac{7}{8} \partial_{\mu} h_{b \nu \sigma \tau} \partial^{\sigma \tau \lambda} \partial_{\lambda} h_{c \rho}+\frac{3}{2} \partial_{\mu} h_{b \nu \sigma \tau} \partial^{\sigma \lambda} \partial_{\lambda \eta} h_{c \rho}{ }^{\tau \eta}-\partial_{\mu} h_{b \nu \sigma \tau} \partial^{\lambda \eta} \partial_{\lambda \eta} h_{c \rho}^{\sigma \tau}+\partial_{\mu} h_{b \nu} \partial_{\lambda} \partial^{\lambda \sigma \tau} h_{c \rho \sigma \tau} \\
& +\frac{7}{4} \partial^{\sigma} h_{b \mu \sigma \tau} \partial^{\tau \lambda \eta} \partial_{\nu} h_{c \rho \lambda \eta}-\frac{9}{8} \partial^{\sigma} h_{b \mu \sigma \tau} \partial^{\tau \lambda} \partial_{\nu \lambda} h_{c \rho}+\frac{1}{4} \partial^{\lambda} h_{b \mu}{ }^{\sigma \tau} \partial_{\nu \sigma \tau \eta} h_{c \rho \lambda}^{\eta}-\frac{3}{4} \partial^{\lambda} h_{b \mu}{ }^{\sigma \tau} \partial_{\nu \sigma \tau \lambda} h_{c \rho} \\
& +2 \partial^{\lambda \tau} h_{b \mu \lambda \sigma} \partial_{\nu \tau \eta} h_{c \rho}^{\sigma \eta}-\frac{1}{4} \partial_{\tau} h_{b \mu \lambda \sigma} \partial_{\nu \eta} \partial^{\lambda \eta} h_{c \rho}^{\sigma \tau}+\frac{3}{4} \partial^{\tau} h_{b \mu \sigma}^{\lambda} \partial_{\nu \lambda \tau \eta} h_{c \rho}^{\sigma \eta}+\partial^{\lambda} h_{b \mu \sigma \tau} \partial_{\nu \lambda \eta} \partial^{\eta} h_{c \rho}^{\sigma \tau} \\
& -\frac{1}{4} \partial^{\sigma \tau} h_{b \mu \sigma \tau} \partial_{\eta} \partial^{\eta \lambda} h_{c \nu \rho \lambda}-\frac{3}{4} \partial^{\sigma} h_{b \mu \sigma \tau} \partial_{\eta} \partial^{\tau \eta \lambda} h_{c \nu \rho \lambda}+\frac{3}{4} \partial^{\lambda} h_{b \mu \sigma \tau} \partial_{\eta} \partial^{\sigma \tau \eta} h_{c \nu \rho \lambda} \\
& +\frac{3}{2} \partial_{\lambda} h_{b \mu \sigma \tau} \partial^{\lambda \sigma \tau \eta} h_{c \nu \rho \eta}-\frac{1}{4} \partial^{\lambda} h_{b \mu} \partial_{\sigma \tau} \partial^{\sigma \tau} h_{c \nu \rho \lambda}+\frac{3}{4} \partial^{\lambda} h_{b \mu \lambda \eta} \partial_{\sigma \tau} \partial^{\sigma \tau} h_{c \nu \rho}^{\eta}+\frac{3}{2} \partial_{\sigma \tau} h_{b \mu \lambda \eta} \partial^{\lambda \sigma \tau} h_{c \nu \rho}^{\eta} \\
& +\frac{1}{3} \partial_{\mu} h_{b \nu \rho \lambda} \partial^{\lambda \sigma \tau \eta} h_{c \sigma \tau \eta}-\frac{15}{4} \partial_{\mu} h_{b \nu \rho \lambda} \partial^{\lambda \sigma \tau} \partial_{\sigma} h_{c \tau}-\frac{11}{4} \partial_{\mu} h_{b \nu \rho \lambda} \partial^{\sigma \tau \eta} \partial_{\sigma} h_{c \tau \eta}^{\lambda} \\
& +\frac{1}{2} \partial_{\mu} h_{b \nu \rho \lambda} \partial^{\sigma \tau} \partial_{\sigma \tau} h_{c}^{\lambda}+\frac{1}{2} \partial_{\eta} h_{b \mu \nu \lambda} \partial^{\lambda} \partial_{\rho \sigma \tau} h_{c}^{\eta \sigma \tau}-\frac{1}{2} \partial_{\eta} h_{b \mu \nu \lambda} \partial^{\lambda \sigma} \partial_{\rho \sigma} h_{c}^{\eta} \\
& -\partial_{\sigma} h_{b \mu \nu \lambda} \partial^{\lambda \sigma} \partial_{\rho \eta} h_{c}^{\eta}-\frac{3}{4} \partial^{\eta} \partial_{\eta} h_{b \mu \nu \lambda} \partial_{\rho \sigma \tau} h_{c}^{\lambda \sigma \tau}+\frac{1}{2} \partial^{\sigma \tau} h_{b \mu \nu \lambda} \partial_{\rho \sigma \tau} h_{c}^{\lambda}+\frac{7}{4} \partial^{\lambda} h_{b \mu \nu \lambda} \partial_{\eta \sigma \tau} \partial^{\eta} h_{c \rho}{ }^{\sigma \tau}
\end{aligned}
$$




$$
\begin{aligned}
& -\frac{1}{4} \partial^{\lambda} h_{b \mu \nu \lambda} \partial_{\sigma \tau} \partial^{\sigma \tau} h_{c \rho}-\frac{3}{2} \partial^{\eta} h_{b \mu \nu \lambda} \partial_{\sigma} \partial^{\lambda \sigma \tau} h_{c \rho \eta \tau}-2 \partial_{\eta} h_{b \mu \nu \lambda} \partial^{\eta \lambda \sigma \tau} h_{c \rho \sigma \tau} \\
& +\frac{1}{2} \partial_{\eta} h_{b \mu \nu \lambda} \partial^{\eta \lambda \sigma} \partial_{\sigma} h_{c \rho}+\frac{1}{4} \partial_{\eta} h_{b \mu \nu \lambda} \partial^{\sigma \tau} \partial_{\sigma \tau} h_{c \rho}^{\eta \lambda}+\frac{1}{2} \partial_{\eta} h_{b \mu \nu \lambda} \partial^{\eta \sigma \tau} \partial_{\sigma} h_{c \rho \tau}^{\lambda}-\frac{1}{4} \partial_{\eta} h_{b \mu \nu \rho} \partial_{\lambda \sigma \tau} \partial^{\lambda} h_{c}^{\eta \sigma \tau} \\
& -\frac{3}{8} \partial_{\eta} h_{b \mu \nu \rho} \partial_{\lambda \sigma} \partial^{\lambda \sigma} h_{c}^{\eta}-\frac{1}{2} \partial_{\eta} h_{b \mu \nu \rho} \partial^{\eta \lambda} \partial_{\lambda \sigma} h_{c}^{\sigma}-\frac{27}{16} \partial_{\mu \nu} h_{b \lambda} \partial^{\lambda \sigma \tau} h_{c \rho \sigma \tau}+\frac{15}{16} \partial_{\mu \nu} h_{b \lambda} \partial^{\lambda \sigma} \partial_{\sigma} h_{c \rho} \\
& -\frac{1}{8} \partial_{\mu \nu} h_{b \lambda} \partial^{\sigma} \partial_{\sigma \eta} h_{c \rho}^{\lambda \eta}+\frac{1}{3} \partial_{\mu} h_{b}^{\lambda \sigma \tau} \partial_{\nu \lambda \sigma \tau} h_{c \rho}+\frac{1}{2} \partial_{\mu \lambda} h_{b}^{\lambda} \partial_{\nu \sigma} \partial^{\sigma} h_{c \rho}-\frac{33}{16} \partial_{\mu} h_{b}^{\lambda} \partial_{\nu \lambda \sigma \tau} h_{c \rho}^{\sigma \tau} \\
& -\frac{23}{4} \partial_{\mu} \partial^{\sigma} h_{b}^{\lambda} \partial_{\nu \lambda \sigma} h_{c \rho}+\frac{5}{8} \partial_{\mu} h_{b}^{\lambda} \partial_{\nu \sigma} \partial^{\sigma \tau} h_{c \rho \lambda \tau}-3 \partial_{\mu} h_{b}^{\lambda \sigma \tau} \partial_{\nu \lambda \eta} \partial^{\eta} h_{c \rho \sigma \tau}-\frac{1}{4} \partial_{\lambda} h_{b}^{\lambda \sigma \tau} \partial_{\mu \nu \sigma \tau} h_{c \rho} \\
& -\frac{3}{2} \partial^{\lambda \sigma} h_{b \lambda} \partial_{\mu \nu} \partial^{\tau} h_{c \rho \sigma \tau}+\frac{11}{4} \partial^{\lambda \sigma} h_{b \lambda} \partial_{\mu \nu \sigma} h_{c \rho}-\frac{15}{16} \partial^{\sigma \tau} h_{b}^{\lambda} \partial_{\mu \nu \lambda} h_{c \rho \sigma \tau}+\frac{43}{16} \partial^{\sigma} \partial_{\sigma} h_{b}^{\lambda} \partial_{\mu \nu \lambda} h_{c \rho} \\
& -\frac{11}{4} \partial^{\sigma \tau} h_{b}^{\lambda} \partial_{\mu \nu \sigma} h_{c \rho \lambda \tau}+\frac{19}{8} \partial^{\sigma} \partial_{\sigma} h_{b}^{\lambda} \partial_{\mu \nu \tau} h_{c \rho \lambda}^{\tau}+\frac{9}{4} \partial_{\eta \lambda} h_{b}^{\eta \sigma \tau} \partial_{\mu \nu \sigma} h_{c \rho \tau}^{\lambda}+\frac{3}{4} \partial_{\eta} h_{b}^{\lambda \sigma \tau} \partial_{\mu \nu \sigma \tau} h_{c \rho \lambda}^{\eta} \\
& +\frac{15}{4} \partial_{\lambda} h_{b}^{\lambda \sigma \tau} \partial_{\mu \nu \eta} \partial^{\eta} h_{c \rho \sigma \tau}-3 \partial^{\eta} h_{b}^{\lambda \sigma \tau} \partial_{\mu \nu \eta \lambda} h_{c \rho \sigma \tau}-\frac{1}{2} \partial_{\mu} h_{b \lambda \sigma \tau} \partial^{\lambda \sigma \tau \eta} h_{c \nu \rho \eta}-\frac{19}{4} \partial_{\mu} h_{b \lambda} \partial^{\lambda \sigma \eta} \partial_{\sigma} h_{c \nu \rho \eta} \\
& +\frac{1}{2} \partial_{\mu} h_{b}^{\lambda} \partial^{\sigma \tau} \partial_{\sigma \tau} h_{c \nu \rho \lambda}-\frac{5}{2} \partial_{\mu} \partial^{\eta} h_{b}^{\lambda \sigma \tau} \partial_{\eta \sigma \tau} h_{c \nu \rho \lambda}-\frac{21}{4} \partial_{\mu} h_{b}^{\lambda \sigma \tau} \partial^{\eta} \partial_{\eta \sigma \tau} h_{c \nu \rho \lambda}+\frac{1}{6} \partial_{\lambda} h_{b}^{\lambda \sigma \tau} \partial_{\mu \sigma \tau} \partial^{\eta} h_{c \nu \rho \eta} \\
& -\frac{1}{2} \partial^{\eta} h_{b}^{\lambda \sigma \tau} \partial_{\mu \lambda \sigma \tau} h_{c \nu \rho \eta}-5 \partial^{\lambda \eta} h_{b \lambda} \partial_{\mu \eta \sigma} h_{c \nu \rho}^{\sigma}-\frac{1}{2} \partial^{\sigma \eta} h_{b}^{\lambda} \partial_{\mu \eta \lambda} h_{c \nu \rho \sigma}-\frac{9}{2} \partial^{\eta} \partial_{\eta} h_{b}^{\lambda} \partial_{\mu \lambda} \partial^{\sigma} h_{c \nu \rho \sigma} \\
& -\frac{1}{2} \partial^{\sigma} \partial_{\sigma} h_{b}^{\lambda} \partial_{\mu \eta} \partial^{\eta} h_{c \nu \rho \lambda}+\partial^{\sigma \tau} h_{b}^{\lambda} \partial_{\mu \sigma \tau} h_{c \nu \rho \lambda}-\frac{11}{2} \partial^{\eta} \partial_{\sigma} h_{b}^{\lambda \sigma \tau} \partial_{\mu \tau \eta} h_{c \nu \rho \lambda}+\frac{3}{4} \partial^{\eta} \partial_{\eta} h_{b}^{\lambda \sigma \tau} \partial_{\mu \sigma \tau} h_{c \nu \rho \lambda} \\
& \left.-\frac{1}{4} \partial_{\lambda} h_{b}^{\lambda \sigma \tau} \partial_{\sigma \tau \eta} \partial^{\eta} h_{c \mu \nu \rho}+\frac{1}{2} \partial^{\eta} h_{b}^{\lambda \sigma \tau} \partial_{\lambda \sigma \tau \eta} h_{c \mu \nu \rho}-\frac{7}{8} \partial_{\lambda} h_{b}^{\lambda} \partial_{\sigma \tau} \partial^{\sigma \tau} h_{c \mu \nu \rho}-\frac{7}{4} \partial^{\sigma \tau} h_{b}^{\lambda} \partial_{\lambda \sigma \tau} h_{c \mu \nu \rho}\right) \\
& +h_{a}^{\mu}\left(\frac{1}{2} \partial_{\mu} h_{b}^{\lambda \sigma \tau} \partial_{\lambda \sigma \tau \rho} h_{c}^{\rho}-\frac{13}{16} \partial_{\mu} h_{b}^{\sigma \tau \lambda} \partial_{\sigma \tau \nu \rho} h_{c \lambda}^{\nu \rho}+\frac{9}{16} \partial_{\mu} h_{b}^{\sigma \tau \lambda} \partial_{\sigma \tau \nu} \partial^{\nu} h_{c \lambda}+\frac{1}{2} \partial_{\mu \lambda} h_{b}^{\lambda \nu \rho} \partial^{\sigma \tau} \partial_{\sigma} h_{c \nu \rho \tau}\right. \\
& -\frac{3}{4} \partial_{\mu} h_{b}^{\lambda \nu \rho} \partial_{\lambda \sigma} \partial^{\sigma \tau} h_{c \nu \rho \tau}+\partial_{\mu} h_{b}^{\lambda \nu \rho} \partial^{\sigma \tau} \partial_{\sigma \tau} h_{c \lambda \nu \rho}+\frac{1}{2} \partial_{\mu} h_{b \lambda} \partial^{\lambda \nu \rho \sigma} h_{c \nu \rho \sigma}-\frac{1}{2} \partial_{\mu} h_{b}^{\lambda} \partial_{\lambda \nu \rho} \partial^{\nu} h_{c}^{\rho} \\
& +\frac{3}{16} \partial_{\mu} h_{b}^{\lambda} \partial^{\nu \rho \sigma} \partial_{\sigma} h_{c \lambda \nu \rho}+\frac{1}{4} \partial_{\mu} h_{b}^{\lambda} \partial^{\rho \sigma} \partial_{\rho \sigma} h_{c \lambda}-\partial_{\lambda} h_{b}^{\lambda \rho \sigma} \partial_{\mu \rho \sigma \tau} h_{c}^{\tau}+\frac{1}{2} \partial_{\tau} h_{b}^{\lambda \rho \sigma} \partial_{\mu \lambda \rho \sigma} h_{c}^{\tau} \\
& +\frac{23}{16} \partial_{\lambda} h_{b}^{\lambda \nu \rho} \partial_{\mu \nu \sigma \tau} h_{c \rho}^{\sigma \tau}-\frac{3}{4} \partial_{\lambda} h_{b}^{\lambda \nu \rho} \partial_{\mu \nu \sigma} \partial^{\sigma} h_{c \rho}-\frac{5}{8} \partial^{\lambda} h_{b}^{\nu \rho \sigma} \partial_{\mu \nu \rho \tau} h_{c \lambda \sigma}^{\tau}+\frac{25}{4} \partial_{\lambda} h_{b}^{\lambda \nu \rho} \partial_{\mu \sigma} \partial^{\sigma \tau} h_{c \nu \rho \tau} \\
& +\partial^{\eta} h_{b}^{\lambda \nu \rho} \partial_{\mu \lambda \sigma} \partial^{\sigma} h_{c \eta \nu \rho}-6 \partial^{\eta} h_{b}^{\lambda \nu \rho} \partial_{\mu \eta \lambda \sigma} h_{c \nu \rho}^{\sigma}-\partial^{\eta} h_{b}^{\lambda \nu \rho} \partial_{\mu \eta \sigma} \partial^{\sigma} h_{c \lambda \nu \rho}-\frac{1}{4} \partial^{\lambda \nu} h_{b \lambda} \partial_{\mu \nu \rho} h_{c}^{\rho} \\
& -\frac{1}{2} \partial_{\nu} h_{b}^{\lambda} \partial_{\mu \lambda \rho \sigma} h_{c}^{\nu \rho \sigma}+\frac{1}{4} \partial^{\rho \nu} h_{b}^{\lambda} \partial_{\mu \lambda \rho} h_{c \nu}-\frac{1}{2} \partial^{\rho} \partial_{\rho} h_{b}^{\lambda} \partial_{\mu \lambda \nu} h_{c}^{\nu}+\frac{5}{4} \partial^{\rho} \partial_{\rho} h_{b}^{\lambda} \partial_{\mu \nu} \partial^{\nu} h_{c \lambda} \\
& -\partial^{\nu \rho} h_{b}^{\lambda} \partial_{\mu \nu \rho} h_{c \lambda}-\frac{5}{12} \partial_{\lambda} h_{b}^{\lambda \nu \rho} \partial_{\nu \rho \sigma \tau} h_{c \mu}^{\sigma \tau}+\frac{1}{3} \partial_{\lambda} h_{b}^{\lambda \nu \rho} \partial_{\nu \rho \sigma} \partial^{\sigma} h_{c \mu}+\frac{2}{3} \partial_{\sigma} h_{b}^{\lambda \nu \rho} \partial_{\lambda \nu \rho \tau} h_{c \mu}^{\sigma \tau} \\
& -\partial^{\sigma} h_{b}^{\lambda \nu \rho} \partial_{\lambda \nu \rho \sigma} h_{c \mu}+\frac{9}{16} \partial_{\lambda} h_{b}^{\lambda} \partial_{\nu \rho \sigma} \partial^{\nu} h_{c \mu}{ }^{\rho \sigma}+\frac{1}{8} \partial_{\lambda} h_{b}^{\lambda} \partial_{\nu \rho} \partial^{\nu \rho} h_{c \mu}-\frac{3}{8} \partial_{\nu} h_{b}^{\lambda} \partial_{\lambda \rho \sigma} \partial^{\sigma} h_{c \mu}{ }^{\nu \rho} \\
& +\frac{3}{8} \partial^{\nu} h_{b}^{\lambda} \partial_{\lambda \nu \rho \sigma} h_{c \mu}^{\rho \sigma}-\frac{1}{4} \partial^{\nu} h_{b}^{\lambda} \partial^{\rho \sigma} \partial_{\rho \sigma} h_{c \mu \nu \lambda}+\frac{1}{4} \partial^{\nu} h_{b}^{\lambda} \partial^{\rho \sigma} \partial_{\nu \rho} h_{c \mu \lambda \sigma}-\frac{1}{8} \partial_{\lambda} h_{b}^{\lambda \nu \rho} \partial^{\sigma \tau} \partial_{\nu \sigma} h_{c \mu \rho \tau} \\
& -\frac{3}{4} \partial^{\lambda} h_{b}^{\nu \rho \sigma} \partial_{\nu \rho \tau} \partial^{\tau} h_{c \mu \lambda \sigma}+2 \partial^{\lambda} h_{b}^{\nu \rho \sigma} \partial_{\lambda \nu \rho \tau} h_{c \mu \sigma}^{\tau}-\frac{1}{4} \partial_{\lambda} h_{b}^{\lambda \rho \sigma} \partial^{\nu \tau} \partial_{\nu \tau} h_{c \mu \rho \sigma}+\frac{1}{2} \partial_{\nu} h_{b}^{\lambda \rho \sigma} \partial^{\nu \tau} \partial_{\lambda \tau} h_{c \mu \rho \sigma} \\
& +\frac{1}{4} \partial^{\nu} h_{b \mu \nu \rho} \partial^{\rho \lambda \sigma \tau} h_{c \lambda \sigma \tau}-\frac{1}{2} \partial^{\lambda} h_{b \mu \nu \rho} \partial^{\nu \rho \sigma \tau} h_{c \lambda \sigma \tau}+\frac{3}{16} \partial^{\lambda} h_{b \mu \nu \rho} \partial^{\nu \rho \sigma} \partial_{\sigma} h_{c \lambda}-\frac{3}{4} \partial_{\nu} h_{b \mu \rho \sigma} \partial^{\nu \rho \sigma \lambda} h_{c \lambda} \\
& +\frac{9}{4} \partial^{\nu \lambda} h_{b \mu \nu \rho} \partial^{\sigma \tau} \partial_{\sigma} h_{c \lambda \tau}^{\rho}+\frac{3}{2} \partial^{\nu \lambda} h_{b \mu \nu \rho} \partial^{\sigma \tau} \partial_{\lambda} h_{c \sigma \tau}^{\rho}+\frac{7}{8} \partial^{\nu} h_{b \mu \nu \rho} \partial^{\lambda \sigma \tau} \partial_{\lambda} h_{c \sigma \tau}^{\rho}-\frac{1}{2} \partial^{\nu} h_{b \mu \nu \rho} \partial_{\sigma \tau} \partial^{\sigma \tau} h_{c}^{\rho} \\
& +\frac{1}{2} \partial_{\lambda} h_{b \mu \nu \rho} \partial_{\sigma \tau} \partial^{\nu \tau} h_{c}^{\lambda \rho \sigma}+\frac{5}{4} \partial_{\lambda} h_{b \mu \nu \rho} \partial^{\nu \lambda} \partial_{\sigma \tau} h_{c}^{\rho \sigma \tau}+\frac{1}{2} \partial_{\lambda} h_{b \mu \nu \rho} \partial^{\nu \lambda \sigma} \partial_{\sigma} h_{c}^{\rho}+\frac{1}{4} \partial_{\lambda} h_{b \mu \nu \rho} \partial_{\sigma \tau} \partial^{\sigma \tau} h_{c}^{\lambda \nu \rho} \\
& -\frac{1}{2} \partial_{\lambda} h_{b \mu \nu \rho} \partial_{\sigma \tau} \partial^{\lambda \tau} h_{c}^{\nu \rho \sigma}+\frac{1}{2} \partial_{\lambda} h_{b \mu} \partial_{\nu \rho \sigma} \partial^{\sigma} h_{c}^{\lambda \nu \rho}+\frac{1}{6} \partial^{\lambda} h_{b \mu} \partial_{\lambda \nu \rho \sigma} h_{c}^{\nu \rho \sigma}+\frac{1}{8} \partial_{\lambda} h_{b \mu} \partial_{\nu \rho} \partial^{\nu \rho} h_{c}^{\lambda} \\
& \left.-\frac{1}{4} \partial^{\lambda} h_{b \mu} \partial_{\lambda \nu \rho} \partial^{\nu} h_{c}^{\rho}\right) \text {. }
\end{aligned}
$$




\section{References}

[1] C. Fronsdal, Phys. Rev. D 18 (1978) 3624.

[2] M. A. Vasiliev, Phys. Lett. B 567 (2003) 139 [hep-th/0304049]; Fortsch. Phys. 52 (2004) 702 [hep-th/0401177]; Comptes Rendus Physique 5 (2004) 1101 [hep-th/0409260].

[3] A. Sagnotti, E. Sezgin and P. Sundell, "On higher spins with a strong $S p(2, \mathbb{R})$ condition," [hep-th/0501156];

X. Bekaert, S. Cnockaert, C. Iazeolla and M.A. Vasiliev, "Nonlinear Higher Spin Theories in Various Dimensions," [hep-th/0503128].

(Published both in the proceedings of the First Solvay Workshop on Higher-Spin Gauge Theories (Brussels, May 2004).)

[4] B. de Wit and D. Z. Freedman, Phys. Rev. D 21 (1980) 358.

[5] A. K. H. Bengtsson, I. Bengtsson and L. Brink, Nucl. Phys. B 227 (1983) 31.

[6] F. A. Berends, G. J. H. Burgers and H. van Dam, Z. Phys. C 24 (1984) 247.

[7] A. K. H. Bengtsson, Phys. Rev. D 32 (1985) 2031.

[8] I. Bengtsson, "A Covariant Three Point Coupling For Spin 3," CERN-TH-4097/85

[9] F. A. Berends, G. J. H. Burgers and H. van Dam, Nucl. Phys. B 260 (1985) 295.

[10] A. K. H. Bengtsson and I. Bengtsson, Class. Quant. Grav. 3 (1986) 927.

[11] F. A. Berends, G. J. H. Burgers and H. van Dam, Nucl. Phys. B 271 (1986) 429.

[12] T. Damour and S. Deser, Class. Quant. Grav. 4 (1987) L95.

[13] A. K. H. Bengtsson, I. Bengtsson and N. Linden, Class. Quant. Grav. 4 (1987) 1333.

[14] A. K. H. Bengtsson, Class. Quant. Grav. 5 (1988) 437.

[15] E. S. Fradkin and R. R. Metsaev, Class. Quant. Grav. 8 (1991) L89;

R. R. Metsaev, Mod. Phys. Lett. A 6 (1991) 359; Mod. Phys. Lett. A 8 (1993) 2413.

[16] R. R. Metsaev, Class. Quant. Grav. 10 (1993) L39; Phys. Lett. B 309 (1993) 39; "Cubic interaction vertices for higher spin fields," in the proceedings of the 2nd International Sakharov Conference on Physics (Moscow, May 1996) [hep-th/9705048]. 
[17] C. Aragone and S. Deser, Phys. Lett. B 86 (1979) 161;

F. A. Berends, J. W. van Holten, B. de Wit and P. van Nieuwenhuizen, J. Phys. A 13 (1980) 1643;

C. Aragone and H. La Roche, Nuovo Cim. A 72 (1982) 149;

S. Deser and Z. Yang, Class. Quant. Grav. 7 (1990) 1491.

[18] E. S. Fradkin and M. A. Vasiliev, Phys. Lett. B 189 (1987) 89; Nucl. Phys. B 291 (1987) 141;

M. A. Vasiliev, Nucl. Phys. B 616 (2001) 106 [Erratum-ibid. B 652 (2003) 407] [hep-th/0106200];

K. B. Alkalaev and M. A. Vasiliev, Nucl. Phys. B 655 (2003) 57 [hep-th/0206068].

[19] S. Weinberg, Phys. Rev. 138 (1965) B988.

[20] G. Barnich and M. Henneaux, Phys. Lett. B 311 (1993) 123 [hep-th/9304057].

[21] M. Henneaux, Contemp. Math. 219 (1998) 93 [hep-th/9712226].

[22] N. Boulanger, T. Damour, L. Gualtieri and M. Henneaux, Nucl. Phys. B 597 (2001) 127 [hep-th/0007220].

[23] X. Bekaert, N. Boulanger and S. Cnockaert, J. Math. Phys. 46 (2005) 012303 [hep-th/0407102].

[24] X. Bekaert and N. Boulanger, Nucl. Phys. B 722 (2005) 225 [hep-th/0505068].

[25] D. Francia and A. Sagnotti, Phys. Lett. B 543 (2002) 303 [hep-th/0207002];

Class. Quant. Grav. 20 (2003) S473 [hep-th/0212185].

[26] D. Francia and A. Sagnotti, Phys. Lett. B 624 (2005) 93 [hep-th/0507144].

[27] A. Pashnev and M. Tsulaia, Mod. Phys. Lett. A 13 (1998) 1853 [hep-th/9803207].

[28] T. Damour and S. Deser, Annales Poincaré Phys. Théor. 47 (1987) 277.

[29] X. Bekaert and N. Boulanger, Phys. Lett. B 561 (2003) 183 [hep-th/0301243]; "Mixed symmetry gauge fields in a flat background," in the proceedings of the International Seminar on Supersymmetries and Quantum Symmetries "SQS 03" (Dubna, July 2003) [hep-th/0310209].

[30] M. Dubois-Violette and M. Henneaux, Lett. Math. Phys. 49 (1999) 245 [math.qa/9907135]; Commun. Math. Phys. 226 (2002) 393 [math.qa/0110088]. 
[31] M. A. Vasiliev, Fortsch. Phys. 36 (1988) 33;

S. E. Konshtein and M. A. Vasiliev, Nucl. Phys. B 312 (1989) 402; Nucl. Phys. B 331 (1990) 475;

M. A. Vasiliev, JHEP 0412 (2004) 046 [hep-th/0404124].

[32] M. A. Vasiliev, Yad. Fiz. 32 (1980) 855.

[33] G. Barnich, F. Brandt and M. Henneaux, Commun. Math. Phys. 174 (1995) 57 [hep-th/9405109].

[34] G. Barnich, N. Bouatta and M. Grigoriev, JHEP 0510, 010 (2005) [hep-th/0507138].

[35] N. Bouatta, "Approche BRST des systèmes invariants de jauge à spins élevés," Master Degree Thesis defended at the Free University of Brussels (Academic year 2003-2004).

[36] G. Barnich, F. Brandt and M. Henneaux, Commun. Math. Phys. 174 (1995) 93 [hep-th/9405194].

[37] J. M. Lee, "Ricci" software package, http://www.math.washington.edu/ lee.

[38] M. Henneaux, B. Knaepen and C. Schomblond, Commun. Math. Phys. 186 (1997) 137 [hep-th/9606181].

[39] M. Henneaux and C. Teitelboim, Quantization of Gauge Systems (Princeton University Press, 1992).

[40] J. A. M. Vermaseren, "New features of FORM," [math-ph/0010025].

[41] V. E. Lopatin and M. A. Vasiliev, Mod. Phys. Lett. A 3 (1988) 257.

[42] N. Boulanger, S. Leclercq and S. Cnockaert, Phys. Rev. D 73 (2006) 065019 [hep-th/0509118]. 